\title{
MAPPING OF NILPOTENT ORBITS UNDER EMBEDDINGS OF REAL FORMS OF EXCEPTIONAL COMPLEX LIE ALGEBRAS *
}

\author{
DRAGOMIR Ž. ĐOKOVIĆ ${ }^{\dagger}$ AND JIRO SEKIGUCHI ${ }^{\ddagger}$
}

\begin{abstract}
We consider Lie algebra monomorphisms $\varphi: \mathfrak{g}_{1} \rightarrow \mathfrak{g}_{2}$ between various noncompact real forms $\mathfrak{g}_{1}, \mathfrak{g}_{2}$ of complex simple Lie algebras $\mathfrak{g}_{1}^{c}, \mathfrak{g}_{2}^{c}$. In all cases that we consider, $\mathfrak{g}_{1}^{c}$ or $\mathfrak{g}_{2}^{c}$ is of exceptional type, with one exception. For each adjoint nilpotent orbit $\mathcal{O}$ of $\mathfrak{g}_{1}$ we determine the adjoint nilpotent orbit of $\mathfrak{g}_{2}$ which contains the image $\varphi(\mathcal{O})$. The adjoint nilpotent orbits of $\mathfrak{g}_{1}$ and $\mathfrak{g}_{2}$ are themselves parametrized by using the Kostant-Sekiguchi correspondence.
\end{abstract}

1. Preliminaries. Let $\mathfrak{g}$ be a semisimple real Lie algebra and $\mathfrak{g}^{c}$ its complexification. Let $\theta$ be a Cartan involution of $\mathfrak{g}$ and $\mathfrak{g}=\mathfrak{k}+\mathfrak{p}$ the corresponding $\mathbf{Z}_{2}$-gradation (a Cartan decomposition). By complexifying, we obtain the $\mathbf{Z}_{2}$-gradation $\mathfrak{g}^{c}=\mathfrak{k}^{c}+\mathfrak{p}^{c}$ and we extend $\theta$ to a complex linear automorphism $\theta^{c}$ of $\mathfrak{g}^{c}$. Denote by $G$ (resp. $G^{c}$ ) the adjoint group of $\mathfrak{g}$ (resp. $\mathfrak{g}^{c}$ ). Thus $G$ is the connected Lie subgroup of $G^{c}$ with Lie algebra $\mathfrak{g}$. The group $G$ (resp. $G^{c}$ ) acts on $\mathfrak{g}$ (resp. $\mathfrak{g}^{c}$ ) via the adjoint action: $(a, x) \rightarrow a \cdot x=\operatorname{Ad}(a)(x)$ where $a \in G^{c}$ (resp. $\left.G\right)$ and $x \in \mathfrak{g}^{c}$ (resp. g). A $G^{c}$ (resp. $G$ )-orbit is nilpotent if it consists of nilpotent elements of $\mathfrak{g}^{c}$ (resp $\mathfrak{g}$ ). There are only finitely many nilpotent $G^{c}$ (resp. $G$ )-orbits in $\mathfrak{g}^{c}$ (resp. $\mathfrak{g}$ ).

Let $K^{c}$ (resp. $K$ ) be the connected Lie subgroup of $G^{c}$ (resp. $G$ ) whose Lie algebra is $\mathfrak{k}^{c}$ (resp. $\mathfrak{k}$ ). By restricting the adjoint action of $G^{c}$, we obtain an action of $K^{c}$ on $\mathfrak{p}^{c}$. The number of nilpotent $K^{c}$-orbits in $\mathfrak{p}^{c}$ is also finite [4].

Let $\mathcal{O}$ be a nilpotent $G^{c}$-orbit in $\mathfrak{g}^{c}$. The intersection $\mathcal{O} \cap \mathfrak{g}$ consists of finitely many connected components $\mathcal{A}_{i}, i=1, \ldots, k$. Moreover, each of these connected components is a single nilpotent $G$-orbit; and $\operatorname{dim}_{\mathbf{R}}\left(\mathcal{A}_{i}\right)=\operatorname{dim}_{\mathbf{C}}(\mathcal{O})$ for each $i$. The intersection $\mathcal{O} \cap \mathfrak{p}^{c}$ also consists of $k$ connected components, say $\mathcal{B}_{i}, i=1, \ldots, k$, each of them is a single nilpotent $K^{c}$-orbit and $\operatorname{dim}_{\mathbf{C}}\left(\mathcal{B}_{i}\right)=\frac{1}{2} \operatorname{dim}_{\mathbf{C}}(\mathcal{O})$ for each $i$. The Kostant-Sekiguchi correspondence (see $[10,4]$ ) establishes a bijection from $\left\{\mathcal{A}_{i}\right\}$ to $\left\{\mathcal{B}_{i}\right\}$.

If $E, H, F \in \mathfrak{g}^{c}$ are nonzero elements satisfying $[H, E]=2 E,[H, F]=-2 F$, and $[F, E]=H$, then we say that $(E, H, F)$ is a standard triple. If moreover $E, F \in \mathfrak{p}^{c}$ and $H \in \mathfrak{k}^{c}$, then we say that $(E, H, F)$ is a normal triple. Let us fix a Cartan subalgebra $\mathfrak{h}$ of $\mathfrak{k}$ and $\tilde{\mathfrak{h}}$ of $\mathfrak{g}$ such that $\tilde{\mathfrak{h}} \supseteq \mathfrak{h}$. Let $\mathfrak{h}^{c}$ and $\tilde{\mathfrak{h}}^{c}$ be their respective complexifications. Let $R$ be the root system of $\left(\mathfrak{k}^{c}, \mathfrak{h}^{c}\right)$, and $\tilde{R}$ that of $\left(\mathfrak{g}^{c}, \tilde{\mathfrak{h}}^{c}\right)$. Finally, let $W$ be the Weyl group of $R, \Pi=\left\{\beta_{1}, \beta_{2}, \ldots\right\}$ a base of $R$ (a system of fundamental roots), and define $\tilde{W}, \tilde{R}$ and $\tilde{\Pi}=\left\{\alpha_{1}, \alpha_{2}, \ldots\right\}$ similarly. If $\tilde{R}$ (resp. $R$ ) is irreducible, we denote its highest root by $\tilde{\alpha}$ (resp. $\tilde{\beta})$.

We say that the $\mathbf{Z}_{2}$-graded Lie algebra $\mathfrak{g}^{c}$ is of inner type if $\mathfrak{k}^{c}$ and $\mathfrak{g}^{c}$ have the same rank, i.e., $\mathfrak{h}^{c}=\tilde{\mathfrak{h}}^{c}$, and otherwise of outer type. In the former case we may view $R$ as a root subsystem of $\tilde{R}$, and we say that the roots in $R$ are compact and the other roots in $\tilde{R}$ are noncompact. In the root diagrams, we shall represent compact (resp. noncompact) roots by black (resp. white) nodes.

\footnotetext{
*Received January 20, 2002; accepted for publication July 4, 2002.

tDepartment of Pure Mathematics, University of Waterloo, Waterloo, Ontario, N2L 3G1, Canada (djokovic@uwaterloo.ca). The author was supported in part by the NSERC Grant A-5285.

$\ddagger$ Department of Mathematics, Tokyo University of Agriculture and Technology, Koganei, Tokyo 184-8588, Japan (sekiguti@cc.tuat.ac.jp). The author was supported by Grant-in-Aid for Scientific Research (No. 13640039), Ministry of Education, Culture, Sports, Science and Technology, Japan.
} 
If $\mathfrak{g}^{c}$ is simple and of inner type, then one can choose a base $\tilde{\Pi}$ of $\tilde{R}$ such that there exists a unique base $\Pi$ of $R$ contained in $\tilde{\Pi} \cup\{-\tilde{\alpha}\}$. We assume that $\tilde{\Pi}$ and $\Pi$ are chosen in this fashion (see Tables 4 and 8).

Let $\mathcal{A}$ be a nonzero nilpotent $K^{c}$-orbit in $\mathfrak{p}^{c}$. We can choose a normal triple $(E, H, F)$ such that $E \in \mathcal{A}, H \in \mathfrak{h}^{c}$, and the numbers $\beta(H)$ are nonnegative integers for each $\beta \in \Pi$. The orbit $\mathcal{A}$ uniquely determines the element $H$ and vice versa. We shall refer to $H$ as the characteristic of the orbit $\mathcal{A}$. Among the algebras that we consider, there are two cases with $\mathfrak{k}^{c}$ non-semisimple. In these two cases $\tilde{\mathfrak{h}}^{c}=\mathfrak{h}^{c}$, $\tilde{\Pi}=\Pi \cup\left\{\beta^{\prime}\right\}$, and we identify $H$ by means of the labels $\beta_{j}(H), \beta_{j} \in \Pi$, and the additional label $\beta^{\prime}(H)$.

The containment relation between the closures of nilpotent $G$-orbits in $\mathfrak{g}$ defines a partial order on the set of these orbits. One obtains similarly a partial order on the set of nilpotent $G^{c}$-orbits in $\mathfrak{g}^{c}$ and the set of nilpotent $K^{c}$-orbits in $\mathfrak{p}^{c}$. We refer to these partial orders as the closure orderings.

It was shown by Barbasch and Sepanski [1] that the Kostant-Sekiguchi correspondence preserves the closure ordering of the two sets of orbits. Let $\mathcal{N}\left(\mathfrak{g}^{c}\right)$ denote the nilpotent variety of $\mathfrak{g}^{c}$ (an irreducible affine variety). We set $\mathcal{N}(\mathfrak{g})=\mathfrak{g} \cap \mathcal{N}\left(\mathfrak{g}^{c}\right)$ and $\mathcal{N}\left(\mathfrak{p}^{c}\right)=\mathfrak{p}^{c} \cap \mathcal{N}\left(\mathfrak{g}^{c}\right)$. If we equip the quotients $\mathcal{N}(\mathfrak{g}) / G$ and $\mathcal{N}\left(\mathfrak{p}^{c}\right) / K^{c}$ with their respective quotient topologies, then the result of Barbasch and Sepanski can be expressed by saying that the Kostant-Sekiguchi correspondence is a homeomorphism between these two finite topological spaces.

We shall use the Cartan notation for the isomorphism types of noncompact real forms of the exceptional complex Lie algebras. Alternatively, these real forms may be distinguished by their Cartan indices $i=\operatorname{dim}(\mathfrak{p})-\operatorname{dim}(\mathfrak{k})$ which are usually written in parentheses.

$$
\begin{aligned}
& E_{6}: \mathrm{EI}=E_{6(6)}, \mathrm{EII}=E_{6(2)}, \mathrm{EIII}=E_{6(-14)}, \mathrm{EIV}=E_{6(-26)} \\
& E_{7}: \mathrm{EV}=E_{7(7)}, \mathrm{EVI}=E_{7(-5)}, \mathrm{EVII}=E_{7(-25)} \\
& E_{8}: \mathrm{EVIII}=E_{8(8)}, \mathrm{EIX}=E_{8(-24)} \\
& F_{4}: \mathrm{FI}=F_{4(4)}, \mathrm{FII}=F_{4(-20)} \\
& G_{2}: \mathrm{GI}=G_{2(2)}
\end{aligned}
$$

Let us now consider two semisimple real Lie algebras, say, $\mathfrak{g}_{1}$ and $\mathfrak{g}_{2}$. The notations $\theta, \theta^{c}, G, G^{c}, W, \Pi$, etc. will be used also for these algebras and the associated groups by adding subscripts 1 or 2 , as appropriate. In particular, $\theta_{1}$ and $\theta_{2}$ are the Cartan involutions of $\mathfrak{g}_{1}$ and $\mathfrak{g}_{2}$, respectively. We say that a Lie algebra monomorphism $\varphi: \mathfrak{g}_{1} \rightarrow \mathfrak{g}_{2}$ is a $\mathbf{Z}_{2}$-embedding of $\mathfrak{g}_{1}$ in $\mathfrak{g}_{2}$ if $\varphi \circ \theta_{1}=\theta_{2} \circ \varphi$. From now on we assume that $\varphi$ is such an embedding. Then $\varphi\left(\mathfrak{k}_{1}\right) \subseteq \mathfrak{k}_{2}$ and $\varphi\left(\mathfrak{p}_{1}\right) \subseteq \mathfrak{p}_{2}$. The complexification $\varphi^{c}: \mathfrak{g}_{1}^{c} \rightarrow \mathfrak{g}_{2}^{c}$ of $\varphi$ will also be called a $\mathbf{Z}_{2}$-embedding. Then we have the commutative diagram:

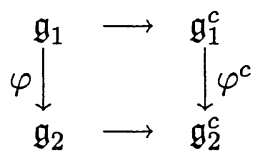

where the horizontal arrows are the inclusion maps. From this diagram we obtain the following commutative diagram for orbit spaces:

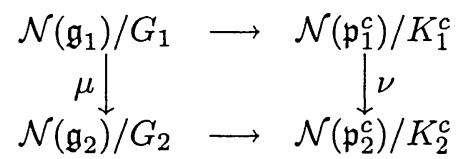


where the horizontal arrows are homeomorphisms given by the Kostant-Sekiguchi correspondence and the vertical arrows $\mu$ and $\nu$ are the continuous maps induced by the $\mathbf{Z}_{2}$-embeddings $\varphi$ and $\varphi^{c}$, respectively.

Our main objective is to give explicit description of the maps $\mu$ and $\nu$ for some interesting $\mathbf{Z}_{2}$-embeddings of real forms of complex simple Lie algebras. These embeddings are taken from an extensive list compiled by Berger [2]. In view of the commutativity of the above diagram, it suffices to determine the map $\nu$. The main results are given in the tables of Sections 2 and 3.

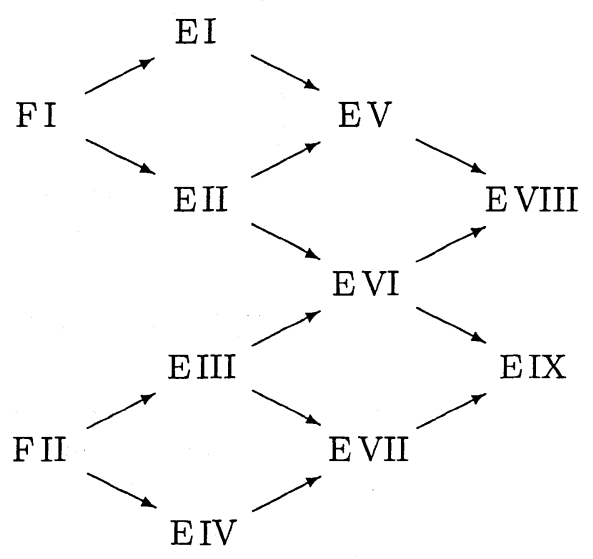

FIGURE 1. Embeddings of real forms

First of all we shall consider the $\mathbf{Z}_{2}$-embeddings shown schematically on Figure 1. We shall describe them in the next section. By analyzing the $\mathbf{Z}_{2}$-embedding EVI $\rightarrow$ E IX and by inspecting the closure diagrams for EVI [7, Figure 2] and EIX [8, Figure 3], we detected an error in the former diagram: The line joining the nodes 22 and 33 should be erased.

In addition to the $\mathbf{Z}_{2}$-embeddings shown on Figure 1 , we shall also consider the following chain of $\mathbf{Z}_{2}$-embeddings of the split real forms:

$$
\mathfrak{s l}(3, \mathbf{R}) \longrightarrow \mathrm{G} \mathrm{I} \longrightarrow \mathfrak{s o}(4,3) \longrightarrow \mathfrak{s o}(4,4) \longrightarrow \mathfrak{s o}(5,4) \longrightarrow \mathrm{F} \mathrm{I}
$$

For each of the arrows in Figure 1 and the diagram (1.3) we describe explicitly the map $\nu$ in tabular form. For the arrows in Figure 1 see Table 2 in the next section, and for those in the diagram (1.3) see the tables in Section 3. In order to make these tables user-friendly, we have included the necessary details about the enumeration of orbits. For the exceptional cases, these details are given in the Appendix.

The second author would like to thank the Department of Pure Mathematics of the University of Waterloo for its hospitality during his visit in September 2001.

2. Embeddings from Figure 1. It is more convenient to work with complex Lie algebras than with the real ones. Hence in order to construct a commutative diagram (1.1) we shall start with the complex $\mathbf{Z}_{2}$-embedding $\varphi^{c}: \mathfrak{g}_{1}^{c} \rightarrow \mathfrak{g}_{2}^{c}$ and then construct the $\mathbf{Z}_{2}$-embedding of real froms $\varphi: \mathfrak{g}_{1} \rightarrow \mathfrak{g}_{2}$ to obtain the diagram (1.1). This is indeed possible by the following result.

Proposition 2.1. Let $\mathfrak{g}_{2}^{c}$ be a semisimple complex Lie algebra and $\mathfrak{g}_{1}^{c}$ a semisimple subalgebra of $\mathfrak{g}_{2}^{c}$. Let $\theta_{2}^{c}$ be an involutorial automorphism of $\mathfrak{g}_{2}^{c}$, such that $\mathfrak{g}_{1}^{c}$ is 
$\theta_{2}^{c}$-stable, and denote by $\theta_{1}^{c}$ the restriction of $\theta_{2}^{c}$ to $\mathfrak{g}_{1}^{c}$. Let $\mathfrak{g}_{i}^{c}=\mathfrak{k}_{i}^{c} \oplus \mathfrak{p}_{i}^{c}$ be the $\mathbf{Z}_{2}$ gradations induced by $\theta_{i}^{c}(i=1,2)$. Then there exist real forms $\mathfrak{g}_{i}$ of $\mathfrak{g}_{i}^{c}$ such that $\mathfrak{g}_{1} \subseteq \mathfrak{g}_{2}$ and $\mathfrak{g}_{i}$ is stable under $\theta_{i}^{c}$. Moreover, the restriction $\theta_{i}=\theta_{i}^{c} \mid \mathfrak{g}_{i}$ is a Cartan involution of $\mathfrak{g}_{i}$. Thus if $\mathfrak{k}_{i}=\mathfrak{g}_{i} \cap \mathfrak{k}_{i}^{c}$ and $\mathfrak{p}_{i}=\mathfrak{g}_{i} \cap \mathfrak{p}_{i}^{c}$, then $\mathfrak{g}_{i}=\mathfrak{k}_{i} \oplus \mathfrak{p}_{i}$ is a Cartan decomposition of $\mathfrak{g}_{i}$.

Proof. Let $U_{1}$ be a maximal compact subgroup of $\operatorname{Aut}\left(\mathfrak{g}_{1}^{c}\right)$ containing $\theta_{1}^{c}$. The Lie algebra $\mathfrak{u}_{1}$ of $U_{1}$ is a compact real form of $\mathfrak{g}_{1}^{c}$ which is invariant under $\theta_{1}^{c}$. Clearly, it is also invariant under $\theta_{2}^{c}$. It follows that $\theta_{2}^{c}$ normalizes the connected compact Lie subgroup $\widehat{U}_{1}$ of $\operatorname{Aut}\left(\mathfrak{g}_{2}^{c}\right)$ having $\mathfrak{u}_{1}$ as its Lie algebra. Consequently, there exists a maximal compact subgroup $U_{2}$ of $\operatorname{Aut}\left(\mathfrak{g}_{2}^{c}\right)$ containing both $\widehat{U}_{1}$ and $\theta_{2}^{c}$. Its Lie algebra, $\mathfrak{u}_{2}$, is a compact real form of $\mathfrak{g}_{2}^{c}$ invariant under $\theta_{2}^{c}$ and such that $\mathfrak{u}_{2} \cap \mathfrak{g}_{1}^{c}=\mathfrak{u}_{1}$. We can now take

$$
\mathfrak{g}_{1}=\left(\mathfrak{u}_{1} \cap \mathfrak{k}_{1}^{c}\right) \oplus i\left(\mathfrak{u}_{1} \cap \mathfrak{p}_{1}^{c}\right), \quad \mathfrak{g}_{2}=\left(\mathfrak{u}_{2} \cap \mathfrak{k}_{2}^{c}\right) \oplus i\left(\mathfrak{u}_{2} \cap \mathfrak{p}_{2}^{c}\right)
$$

We may (and we do) assume that our $\mathbf{Z}_{2}$-embeddings $\varphi^{c}$ are such that $\varphi^{c}\left(\mathfrak{h}_{1}^{c}\right) \subseteq \mathfrak{h}_{2}^{c}$ and $\varphi^{c}\left(\tilde{\mathfrak{h}}_{1}^{c}\right) \subseteq \tilde{\mathfrak{h}}_{2}^{c}$.

Assume that $\mathfrak{g}_{1}^{c}$ and $\mathfrak{g}_{2}^{c}$ are of inner type. Then we say that an embedding of root systems $\tilde{R}_{1} \rightarrow \tilde{R}_{2}$ is a $\mathbf{Z}_{2}$-embedding if the compact roots are mapped to compact and noncompact to noncompact. Such embedding is uniquely determined by its restriction to a base of $\tilde{R}_{1}$. It is easy to see that every $\mathbf{Z}_{2}$-embedding $\tilde{R}_{1} \rightarrow \tilde{R}_{2}$ can be lifted to a $\mathbf{Z}_{2}$-embedding $\mathfrak{g}_{1}^{c} \rightarrow \mathfrak{g}_{2}^{c}$.

We describe our embeddings in Table 1 . In order to be able to distinguish the roots in $R_{1}$ or $\tilde{R}_{1}$ from those in $R_{2}$ or $\tilde{R}_{2}$, we shall use the superscript ${ }^{(1)}$ for the former and ${ }^{(2)}$ for the latter. We write $\beta_{i}^{(1)} \rightarrow \beta_{j}^{(2)}$ if $\varphi^{c}$ maps the $\beta_{i}^{(1)}$-root space of $\mathfrak{g}_{1}^{c}$ into the $\beta_{j}^{(2)}$-root space of $\mathfrak{g}_{2}^{c}$. Similarly, we write $\beta_{i}^{(1)} \rightarrow\left\{\beta_{j}^{(2)}, \beta_{k}^{(2)}\right\}$ if $\varphi^{c}$ embeds the $\beta_{i}^{(1)}$-root space of $\mathfrak{g}_{1}^{c}$ diagonally into the sum of the root spaces of $\mathfrak{g}_{2}^{c}$ corresponding to the roots $\beta_{j}^{(2)}$ and $\beta_{k}^{(2)}$. In some cases we give only the restriction of $\varphi^{c}$ which embeds $\mathfrak{k}_{1}^{c}$ into $\mathfrak{k}_{2}^{c}$. For our choice of the simple roots of $\tilde{R}$ and $R$ see Table 8 in the Appendix. 
TABLE 1. Description of the $\mathbf{Z}_{2}$-embeddings from Figure 1

\begin{tabular}{|c|c|}
\hline $\mathrm{F} \mathrm{I} \rightarrow \mathrm{E} \mathrm{I}$ & $\beta_{k}^{(1)} \rightarrow \beta_{k+1}^{(2)}, k=1,2,3 ; \beta_{4}^{(1)} \rightarrow-\tilde{\beta}^{(2)}$ \\
\hline $\mathrm{F} \mathrm{I} \rightarrow \mathrm{E}$ II & $\begin{array}{l}\beta_{1}^{(1)} \rightarrow\left\{\beta_{1}^{(2)}, \beta_{5}^{(2)}\right\}, \beta_{2}^{(1)} \rightarrow\left\{\beta_{2}^{(2)}, \beta_{4}^{(2)}\right\} \\
\beta_{3}^{(1)} \rightarrow \beta_{3}^{(2)}, \beta_{4}^{(1)} \rightarrow \beta_{6}^{(2)}\end{array}$ \\
\hline $\mathrm{F} \mathrm{II} \rightarrow \mathrm{E}$ III & $\beta_{k}^{(1)} \rightarrow \beta_{k}^{(2)}, k=1,2,3 ; \beta_{4}^{(1)} \rightarrow\left\{\beta_{4}^{(2)}, \beta_{5}^{(2)}\right\}$ \\
\hline $\mathrm{F} \mathrm{II} \rightarrow \mathrm{E} \mathrm{IV}$ & $\beta_{1}^{(1)} \rightarrow-\tilde{\beta}^{(2)} ; \beta_{k}^{(1)} \rightarrow \beta_{k-1}^{(2)}, k=2,3,4$ \\
\hline $\mathrm{EI} \rightarrow \mathrm{EV}$ & $\beta_{k}^{(1)} \rightarrow\left\{\beta_{k}^{(2)}, \beta_{8-k}^{(2)}\right\}, k=1,2,3 ; \beta_{4}^{(1)} \rightarrow \beta_{4}^{(2)}$ \\
\hline $\mathrm{E} \mathrm{II} \rightarrow \mathrm{E} \mathrm{V}$ & $\begin{array}{l}\beta_{k}^{(1)} \rightarrow \beta_{k}^{(2)}, 1 \leq k \leq 5 ; \beta_{6}^{(1)} \rightarrow \beta_{7}^{(2)} \\
\alpha_{2}^{(1)} \rightarrow \alpha_{2}^{(2)}+\alpha_{4}^{(2)}+\alpha_{5}^{(2)}+\alpha_{6}^{(2)}\end{array}$ \\
\hline $\mathrm{E} \mathrm{II} \rightarrow \mathrm{E} \mathrm{VI}$ & $\begin{array}{l}\beta_{k}^{(1)} \rightarrow \beta_{k}^{(2)}, 1 \leq k \leq 5 ; \beta_{6}^{(1)} \rightarrow \beta_{7}^{(2)} \\
\alpha_{2}^{(1)} \rightarrow \alpha_{2}^{(2)}+\alpha_{4}^{(2)}+\alpha_{5}^{(2)}+\alpha_{6}^{(2)}\end{array}$ \\
\hline $\mathrm{E} \mathrm{III} \rightarrow \mathrm{E} \mathrm{VI}$ & $\beta_{k}^{(1)} \rightarrow \beta_{k+1}^{(2)}, 1 \leq k \leq 5 ; \beta_{6}^{(1)} \rightarrow \alpha_{6}^{(2)}$ \\
\hline $\mathrm{E} \mathrm{III} \rightarrow \mathrm{E}$ VII & $\begin{array}{l}\beta_{1}^{(1)} \rightarrow \beta_{1}^{(2)}, \beta_{k}^{(1)} \rightarrow \beta_{k+1}^{(2)}, k=2,3,4 \\
\beta_{5}^{(1)} \rightarrow \beta_{2}^{(2)}, \beta_{6}^{(1)} \rightarrow \alpha_{6}^{(2)}+\alpha_{7}^{(2)}\end{array}$ \\
\hline $\mathrm{E} \mathrm{IV} \rightarrow \mathrm{E}$ VII & $\begin{array}{l}\beta_{1}^{(1)} \rightarrow \beta_{2}^{(2)}, \beta_{2}^{(1)} \rightarrow \beta_{4}^{(2)}, \beta_{3}^{(1)} \rightarrow\left\{\beta_{3}^{(2)}, \beta_{5}^{(2)}\right\} \\
\beta_{4}^{(1)} \rightarrow\left\{\beta_{1}^{(2)}, \beta_{6}^{(2)}\right\}\end{array}$ \\
\hline $\mathrm{E} \mathrm{V} \rightarrow \mathrm{E} \mathrm{VIII}$ & $\begin{array}{l}\beta_{k}^{(1)} \rightarrow \beta_{k}^{(2)}, 1 \leq k \leq 7 \\
\alpha_{2}^{(1)} \rightarrow \alpha_{1}^{(2)}+\alpha_{2}^{(2)}+2 \alpha_{3}^{(2)}+2 \alpha_{4}^{(2)}+\alpha_{5}^{(2)}\end{array}$ \\
\hline $\mathrm{E} \mathrm{VI} \rightarrow \mathrm{E}$ VIII & $\begin{array}{l}\beta_{k}^{(1)} \rightarrow \beta_{k}^{(2)}, 1 \leq k \leq 4 ; \beta_{5}^{(1)} \rightarrow \beta_{8}^{(2)} \\
\beta_{6}^{(1)} \rightarrow \beta_{7}^{(2)}, \beta_{7}^{(1)} \rightarrow \beta_{1}^{(2)} \\
\alpha_{6}^{(1)} \rightarrow \alpha_{1}^{(2)}+\alpha_{3}^{(2)}+\alpha_{4}^{(2)}+\alpha_{5}^{(2)}+\alpha_{6}^{(2)}+\alpha_{7}^{(2)}+\alpha_{8}^{(2)}\end{array}$ \\
\hline $\mathrm{E} \mathrm{VI} \rightarrow \mathrm{E} \mathrm{IX}$ & $\begin{array}{l}\beta_{k}^{(1)} \rightarrow \beta_{8-k}^{(2)}, 1 \leq k \leq 4 ; \beta_{5}^{(1)} \rightarrow \beta_{2}^{(2)} \\
\beta_{6}^{(1)} \rightarrow \beta_{3}^{(2)}, \beta_{7}^{(1)} \rightarrow \beta_{8}^{(2)} \\
\alpha_{6}^{(1)} \rightarrow \alpha_{1}^{(2)}+\alpha_{3}^{(2)}+\alpha_{4}^{(2)}+\alpha_{5}^{(2)}+\alpha_{6}^{(2)}+\alpha_{7}^{(2)}+\alpha_{8}^{(2)}\end{array}$ \\
\hline $\mathrm{E}$ VII $\rightarrow$ E IX & $\beta_{k}^{(1)} \rightarrow \beta_{k}^{(2)}, 1 \leq k \leq 6 ; \beta_{7}^{(1)} \rightarrow \alpha_{7}^{(2)}+\alpha_{8}^{(2)}$ \\
\hline
\end{tabular}

Let $\mathcal{O}_{1}^{i} \subseteq \mathfrak{p}_{1}^{c}$ be the $i$-th nonzero nilpotent $K_{1}^{c}$-orbit and let $H_{1}^{i} \in \mathfrak{h}_{1}^{c}$ be its characteristic. Define similarly $\mathcal{O}_{2}^{j} \subseteq \mathfrak{p}_{2}^{c}$ and $H_{2}^{j} \in \mathfrak{h}_{2}^{c}$. We shall write $i \rightarrow j$ if $\varphi^{c}\left(\mathcal{O}_{1}^{i}\right) \subseteq \mathcal{O}_{2}^{j}$.

TheOREM 2.2. Consider the $\mathbf{Z}_{2}$-embeddings $\varphi^{c}: \mathfrak{g}_{1}^{\mathfrak{c}} \rightarrow \mathfrak{g}_{2}^{c}$ from Figure 1 and described by Table 1. Then the nonzero nonempty fibers of the map $\nu$ (see the diagram (1.2)) are as given in Table 2.

In Table 2, for each value of $j \neq 0$, we have recorded the superscripts $i$ (if any) 
such that $i \rightarrow j$. The $i$ 's are listed first (on the left hand side) and then the $j$ (on the right hand side). For instance, we have $11,12 \rightarrow 7$ under the embedding FI $\rightarrow$ EI. The arrows are omitted. If $i \rightarrow j$ then, in general, $\varphi^{c}\left(H_{1}^{i}\right)$ is not equal to $H_{2}^{j}$, but they belong to the same orbit of the Weyl group $W_{2}$.

The above theorem is a simple consequence of the following proposition. Indeed the labels of $\varphi^{c}\left(H_{1}^{i}\right)$ can be computed by using the transformation rules given in Table 3 , and then, by using the action of $W_{2}$, one can determine the superscript $j$ such that $i \rightarrow j$.

Proposition 2.3. Consider the $\mathbf{Z}_{2}$-embeddings $\varphi^{c}: \mathfrak{g}_{1}^{c} \rightarrow \mathfrak{g}_{2}^{c}$ from Figure 1 and described by Table 1. Let $\mathcal{O}_{1}^{i} \subseteq \mathfrak{p}_{1}^{c}$ and $\mathcal{O}_{2}^{j} \subseteq \mathfrak{p}_{2}^{c}$ be nonzero nilpotent orbits such that $\varphi^{c}\left(\mathcal{O}_{1}^{i}\right) \subseteq \mathcal{O}_{2}^{j}$. If $H_{1}^{i} \in \mathfrak{h}_{1}^{c}$ is the characteristic of the orbit $\mathcal{O}_{1}^{i}$, then the labels $\beta_{k}^{(2)}\left(\varphi^{c} H_{1}^{i}\right)$ of the element $\varphi^{c}\left(H_{1}^{i}\right) \in \mathfrak{h}_{2}^{c}$ can be computed from the labels $\beta_{k}^{(1)}\left(H_{1}^{i}\right)$ by using the transformation rules given in Table 3.

Proof. The proofs are different in each case but they are of similar nature. We shall give the details for four cases only.

We derive first the transformation rule for the $\mathbf{Z}_{2}$-embedding $\mathrm{FI} \rightarrow \mathrm{EI}$. Thus $\mathfrak{g}_{1}$ is of type FI. The labels of $H_{1}^{i}$ are given in column 2 of Table 3 as " $a b c d$ ". Using the first row of Table 1, this means that

$$
\begin{aligned}
& \beta_{2}^{(2)}\left(\varphi^{c} H_{1}^{i}\right)=\beta_{1}^{(1)}\left(H_{1}^{i}\right)=a, \quad \beta_{3}^{(2)}\left(\varphi^{c} H_{1}^{i}\right)=\beta_{2}^{(1)}\left(H_{1}^{i}\right)=b, \\
& \beta_{4}^{(2)}\left(\varphi^{c} H_{1}^{i}\right)=\beta_{3}^{(1)}\left(H_{1}^{i}\right)=c, \quad-\tilde{\beta}^{(2)}\left(\varphi^{c} H_{1}^{i}\right)=\beta_{4}^{(1)}\left(H_{1}^{i}\right)=d .
\end{aligned}
$$

Since $\tilde{\beta}^{(2)}=2 \beta_{1}^{(2)}+2 \beta_{2}^{(2)}+2 \beta_{3}^{(2)}+\beta_{4}^{(2)}$, we have $-d=2 x+2 a+2 b+c$ where $x=\beta_{1}^{(2)}\left(\varphi^{c} H_{1}^{i}\right)$. This gives the required formula for the label $x$.

Next, we consider the embedding F I $\rightarrow$ EII. Let $\sigma$ be the diagram automorphism of $\mathfrak{g}_{2}^{c}$ of order two which fixes the root spaces for $\alpha_{2}^{(2)}$ and $\alpha_{4}^{(2)}$ and interchanges those for $\alpha_{1}^{(2)}$ and $\alpha_{6}^{(2)}$ as well as those for $\alpha_{3}^{(2)}$ and $\alpha_{5}^{(2)}$ (see e.g. [3, Chapter 8, $\S 5$, Exercise 13]). The fixed point subalgebra of $\sigma$ is a simple Lie algebra of type $F_{4}$ which we can identify with our $\mathfrak{g}_{1}^{c}$, and so we take $\varphi_{1}^{c}$ to be the inclusion map. The automorphism $\theta_{2}^{c}$ of $\mathfrak{g}_{2}^{c}$ defined by the $\mathbf{Z}_{2}$-gradation of $\mathfrak{g}_{2}^{c}$ exhibited in Table 8 (with $\mathfrak{k}_{2}^{c}$ of type $A_{5}+A_{1}$ ) leaves $\mathfrak{g}_{1}^{c}$ invariant. We denote by $\theta_{1}^{c}$ the restriction of $\theta_{2}^{c}$ to $\mathfrak{g}_{1}^{c}$. Then $\mathfrak{k}_{1}^{c}$ is of type $C_{3}+A_{1}$. By Proposition 2.1 this gives a $\mathbf{Z}_{2}$-embedding FI $\rightarrow$ EII. The Cartan subalgebra $\mathfrak{h}_{1}^{c}$ is the subspace of $\mathfrak{h}_{2}^{c}$ defined by the equations $\alpha_{1}^{(2)}(H)=\alpha_{6}^{(2)}(H)$ and $\alpha_{3}^{(2)}(H)=\alpha_{5}^{(2)}(H)$. If $i \rightarrow j$ and the labels of $H_{1}^{i}$ are " $a b c d$ ", i.e.,

$$
\beta_{1}^{(1)}\left(H_{1}^{i}\right)=a, \quad \beta_{2}^{(1)}\left(H_{1}^{i}\right)=b, \quad \beta_{3}^{(1)}\left(H_{1}^{i}\right)=c, \quad \beta_{4}^{(1)}\left(H_{1}^{i}\right)=d,
$$

then the labels of $H_{2}^{j}=H_{1}^{i}$ are given by " $a b c b a d$ ". This follows from the fact that

$$
\begin{aligned}
& \left.\beta_{1}^{(2)}\right|_{\mathfrak{h}_{1}^{c}}=\beta_{5}^{(2)}\left|\mathfrak{h}_{1}^{c}=\beta_{1}^{(1)}, \quad \beta_{3}^{(2)}\right|_{\mathfrak{h}_{1}^{c}}=\beta_{3}^{(1)}, \\
& \left.\beta_{2}^{(2)}\right|_{\mathfrak{h}_{1}^{c}}=\beta_{4}^{(2)}\left|\mathfrak{h}_{1}^{c}=\beta_{2}^{(1)}, \quad \beta_{6}^{(2)}\right|_{\mathfrak{h}_{1}^{c}}=\beta_{4}^{(1)} .
\end{aligned}
$$

We shall now derive the transformation rules for the $\mathbf{Z}_{2}$-embeddings $\mathrm{E}$ III $\rightarrow \mathrm{E}$ VI and EIII $\rightarrow$ E VII. Thus $\mathfrak{g}_{1}$ is of type EIII. The labels of $H_{1}^{i}$ are given in the second 
TABLE 2. Mapping of nilpotent orbits

\begin{tabular}{|c|c|c|c|c|c|c|c|c|c|}
\hline \multicolumn{10}{|c|}{$\mathrm{F} \mathrm{I} \rightarrow \mathrm{E} \mathrm{I}$} \\
\hline 1 & 1 & 2,3 & 2 & 4,5 & 3 & 8 & 4 & 6,7 & 5 \\
\hline 9 & 6 & 11,12 & 7 & 10 & 10 & 13 & 11 & 18 & 12 \\
\hline 19,20 & 13 & 21 & 14 & 14,15 & 15 & 23 & 19 & 26 & 20 \\
\hline 24,25 & 21 & 22 & 22 & 16,17 & 23 & & & & \\
\hline \multicolumn{10}{|c|}{$\mathrm{F} \mathrm{I} \rightarrow \mathrm{E}$ II } \\
\hline 1 & 1 & 2 & 2 & 3 & 3 & 4 & 4 & 5 & 5 \\
\hline 6 & 6 & 7 & 7 & 8 & 8 & 9 & 11 & 10 & 14 \\
\hline 11 & 15 & 12 & 16 & 13 & 17 & 14 & 18 & 15 & 19 \\
\hline 16 & 20 & 17 & 21 & 18 & 22 & 19 & 23 & 20 & 24 \\
\hline 21 & 31 & 23 & 32 & 22 & 33 & 24 & 34 & 25 & 35 \\
\hline 26 & 37 & & & & & & & & \\
\hline \multicolumn{10}{|c|}{ F II $\rightarrow$ E III } \\
\hline 1 & 5 & 2 & 9 & & & & & & \\
\hline \multicolumn{10}{|c|}{$\mathrm{F} \mathrm{II} \rightarrow \mathrm{E} \mathrm{IV}$} \\
\hline 1 & 1 & 2 & 2 & & & & & & \\
\hline \multicolumn{10}{|c|}{$\mathrm{E} \mathrm{I} \rightarrow \mathrm{EV}$} \\
\hline 1 & 1 & 2 & 2 & 3 & 5 & 5 & 6 & 4 & 7 \\
\hline 8 & 12 & 10 & 15 & 7 & 20 & 6 & 21 & 11 & 24 \\
\hline 15 & 25 & 12 & 26 & 23 & 27 & 13 & 30 & 9 & 43 \\
\hline 16 & 50 & 17 & 53 & 14 & 59 & 19 & 62 & 22 & 63 \\
\hline 21 & 66 & 18 & 81 & 20 & 84 & & & & \\
\hline \multicolumn{10}{|c|}{$\mathrm{E} \mathrm{II} \rightarrow \mathrm{EV}$} \\
\hline 1 & 1 & 2,3 & 2 & 4,5 & 5 & $\overline{6,7}$ & 6 & 8 & 7 \\
\hline 9 & 10 & 10 & 11 & 12 & 13 & 13 & 14 & 14 & 15 \\
\hline 15,16 & 20 & 11 & 21 & 17 & 24 & 18,19 & 25 & 22 & 26 \\
\hline 20,21 & 27 & 23,24 & 30 & 25,26 & 38 & 28 & 48 & 27 & 49 \\
\hline 29 & 51 & 30 & 52 & 31 & 59 & 32 & 62 & 33 & 63 \\
\hline 34,35 & 66 & 36 & 80 & 37 & 84 & & & & \\
\hline \multicolumn{10}{|c|}{$\mathrm{E} \mathrm{II} \rightarrow \mathrm{E} \mathrm{VI}$} \\
\hline 1 & 1 & 2 & 2 & 3 & 3 & 4 & 4 & 5 & 5 \\
\hline 6 & 6 & 8 & 7 & 7 & 8 & 9,10 & 9 & 12,13 & 10 \\
\hline 14 & 11 & 15 & 12 & 16 & 13 & 11 & 15 & 17 & 16 \\
\hline 18 & 17 & 19 & 18 & 20 & 19 & 21 & 20 & 22 & 21 \\
\hline 23 & 22 & 24 & 23 & 25 & 25 & 26 & 26 & 27,28 & 27 \\
\hline 29,30 & 28 & 31 & 30 & 32 & 31 & 33 & 32 & 34 & 33 \\
\hline 35 & 34 & 36 & 36 & 37 & 37 & & & & \\
\hline \multicolumn{10}{|c|}{$\mathrm{E}$ III $\rightarrow$ E VI } \\
\hline 1,2 & 1 & 3,4 & 2 & 5 & 3 & 6 & 8 & 7,8 & 9 \\
\hline 10,11 & 13 & 9 & 14 & 12 & 26 & & & & \\
\hline \multicolumn{10}{|c|}{$\mathrm{E}$ III $\rightarrow$ E VII } \\
\hline 1 & 1 & 2 & 2 & 3 & 3 & 4 & 4 & 5 & 5 \\
\hline 6 & 10 & 7 & 11 & $\varepsilon$ & 12 & 10 & 13 & 11 & 14 \\
\hline 9 & 15 & 12 & 20 & & & & & & \\
\hline
\end{tabular}


TABLE 2. (continued)

\begin{tabular}{|c|c|c|c|c|c|c|c|c|c|}
\hline \multicolumn{10}{|c|}{$\mathrm{E} I V \rightarrow \mathrm{E}$ VII } \\
\hline 1 & 5 & 2 & 15 & & & & & & \\
\hline \multicolumn{10}{|c|}{$\mathrm{E} V \rightarrow \mathrm{E}$ VIII } \\
\hline 1 & 1 & 2 & 2 & $3,4,5$ & 3 & 6 & 4 & 7 & 5 \\
\hline 8,9 & 6 & 10,11 & 7 & 12 & 8 & 13,14 & 9 & 15 & 10 \\
\hline 20 & 11 & 16,17 & 12 & 18,19 & 13 & 21 & 16 & 24 & 17 \\
\hline $22,23,25$ & 18 & 26 & 19 & 27 & 20 & 30 & 21 & 28,29 & 23 \\
\hline 31,32 & 24 & 33,34 & 25 & 35,36 & 27 & 37 & 28 & 38 & 29 \\
\hline 43 & 30 & 39,40 & 31 & 41,42 & 32 & 46,47 & 33 & 48,49 & 37 \\
\hline 50 & 38 & 51,52 & 40 & 53 & 41 & 54 & 46 & $44,45,59$ & 47 \\
\hline 55,56 & 48 & 57,58 & 49 & 62 & 54 & 63 & 55 & 66 & 56 \\
\hline 60,61 & 58 & 64,65 & 61 & 69,70 & 64 & 67,68 & 65 & 74,75 & 66 \\
\hline 71 & 71 & 72,73 & 73 & 76,77 & 75 & 78,79 & 76 & 80 & 77 \\
\hline 81 & 78 & 84 & 82 & 82,83 & 83 & 85,86 & 89 & 87,88 & 90 \\
\hline 89,90 & 97 & 91,92 & 103 & 93,94 & 108 & & & & \\
\hline \multicolumn{10}{|c|}{$\mathrm{E} V \mathrm{VI} \rightarrow \mathrm{E}$ VIII } \\
\hline 1 & 1 & 2,3 & 2 & 4,5 & 3 & 6,8 & 4 & 7 & 5 \\
\hline 9 & 7 & 10 & 9 & 11 & 10 & 12,13 & 11 & 14 & 14 \\
\hline 15 & 16 & 16 & 17 & 17,18 & 18 & 21 & 19 & 19,20 & 20 \\
\hline 22,23 & 21 & 24 & 26 & 25,26 & 29 & 27 & 37 & 28 & 40 \\
\hline 29 & 45 & 30 & 47 & 31 & 54 & 32 & 55 & 33,34 & 56 \\
\hline 35 & 70 & 36 & 77 & 37 & 102 & & & & \\
\hline \multicolumn{10}{|c|}{$\mathrm{E} \mathrm{VI} \rightarrow \mathrm{E} \mathrm{IX}$} \\
\hline 1 & 1 & 2 & 2 & 3 & 3 & 4 & 4 & 5 & 5 \\
\hline 6 & 6 & 7 & 7 & 8 & 8 & 9 & 9 & 11 & 10 \\
\hline 10 & 11 & 12 & 12 & 13 & 13 & 14,15 & 14 & 16 & 15 \\
\hline 18 & 16 & 17 & 17 & 20 & 18 & 19 & 19 & 21 & 20 \\
\hline 22 & 21 & 23 & 22 & 24 & 23 & 25 & 24 & 26 & 25 \\
\hline 27 & 26 & 28 & 27 & 29 & 28 & 30 & 29 & 31 & 30 \\
\hline 32 & 31 & 33 & 32 & 34 & 33 & 35 & 34 & 36 & 35 \\
\hline 37 & 36 & & & & & & & & \\
\hline \multicolumn{10}{|c|}{$\mathrm{E}$ VII $\rightarrow$ E IX } \\
\hline 1,2 & 1 & 3,4 & 2 & 5 & 3 & 6,7 & 4 & 8,9 & 5 \\
\hline 10 & 8 & 11,12 & 9 & 13,14 & 13 & 15 & 14 & 16,19 & 16 \\
\hline 17,18 & 17 & 20 & 25 & 21,22 & 29 & & & & \\
\hline
\end{tabular}

column of Table 3 as "abcdef". This means that

$$
\begin{aligned}
& \beta_{1}^{(1)}\left(H_{1}^{i}\right)=a, \quad \beta_{2}^{(1)}\left(H_{1}^{i}\right)=b, \quad \beta_{3}^{(1)}\left(H_{1}^{i}\right)=c, \\
& \beta_{4}^{(1)}\left(H_{1}^{i}\right)=d, \quad \beta_{5}^{(1)}\left(H_{1}^{i}\right)=e, \quad \beta_{6}^{(1)}\left(H_{1}^{i}\right)=f
\end{aligned}
$$

(see the diagram for $\mathfrak{g}=\mathrm{EIII}$ in Table 8 for the definition of the $\beta_{j}$ 's). The fundamental weights of $\left(\mathfrak{g}_{2}^{c}, \mathfrak{h}_{2}^{c}\right)$ will be denoted by $\omega_{k}^{(2)}, 1 \leq k \leq 7$. 
TABLE 3. Transformation rules for $\mathbf{Z}_{2}$-embeddings in Figure 1

\begin{tabular}{|c|c|c|c|}
\hline $\mathfrak{g}_{1} \rightarrow \mathfrak{g}_{2}$ & $H_{1}^{i}$ & $\varphi^{c}\left(H_{1}^{i}\right)$ & $x, y, z$ \\
\hline $\begin{array}{l}\mathrm{F} \mathrm{I} \rightarrow \mathrm{E} \mathrm{I} \\
\mathrm{F} \mathrm{I} \rightarrow \mathrm{E} \mathrm{II}\end{array}$ & $a b c d$ & $\begin{array}{l}x a b c \\
a b c b a d\end{array}$ & $x=-(a+b)-\frac{1}{2}(c+d)$ \\
\hline $\begin{array}{l}\text { F II } \rightarrow \text { E III } \\
\text { F II } \rightarrow \text { E IV }\end{array}$ & $a b c d$ & $\begin{array}{l}a b c d d x \\
b c d x\end{array}$ & $x=-(b+2 d)-\frac{1}{2}(a+3 c)$ \\
\hline $\mathrm{E} \mathrm{I} \rightarrow \mathrm{E} \mathrm{V}$ & $a b c d$ & $a b c d c b a$ & \\
\hline $\begin{array}{l}\mathrm{E} \mathrm{II} \rightarrow \mathrm{E} \mathrm{V} \\
\mathrm{E} \mathrm{II} \rightarrow \mathrm{E} \mathrm{VI}\end{array}$ & $a b c d e f$ & $\begin{array}{l}\text { abcdexf } \\
a b c d e y f\end{array}$ & $\begin{array}{l}x=-\frac{1}{6}(a+2 b+3 c+4 d+5 e+3 f) \\
y=e+f+2 x\end{array}$ \\
\hline $\begin{array}{l}\mathrm{E} \text { III } \rightarrow \text { E VI } \\
\mathrm{E} \mathrm{III} \rightarrow \mathrm{E} \mathrm{VII}\end{array}$ & abcde $f$ & $\begin{array}{l}\text { yabcde } x \\
\text { aebcdz-x }\end{array}$ & $\begin{array}{l}x=-2 c-e-\frac{1}{3}(2 a+4 b+5 d+4 f) \\
y=-2 c-e-\frac{1}{3}(4 a+5 b+4 d+2 f) \\
z=f+x\end{array}$ \\
\hline $\mathrm{E} \mathrm{IV} \rightarrow \mathrm{E}$ VII & $a b c d$ & $d a c b c d x$ & $x=-(a+2 b+3 c+2 d)$ \\
\hline $\mathrm{E} \mathrm{V} \rightarrow \mathrm{E}$ VIII & $a b c d e f g$ & $a b c d e f x g$ & $\begin{array}{l}x=-d \\
\quad-\frac{1}{4}(a+2 b+3 c+5 e+6 f+3 g)\end{array}$ \\
\hline $\begin{array}{l}\mathrm{E} \mathrm{VI} \rightarrow \mathrm{E} \mathrm{VIII} \\
\mathrm{E} \mathrm{VI} \rightarrow \mathrm{E} \mathrm{IX}\end{array}$ & $a b c d e f g$ & $\begin{array}{l}\text { gxabcdfe } \\
\text { yefdcba } g\end{array}$ & $\begin{array}{l}x=-(a+b+c+d)-\frac{1}{2}(e+f+g) \\
y=-(b+2 d+e)-\frac{1}{2}(a+3 c+3 f)\end{array}$ \\
\hline $\mathrm{E} \mathrm{VII} \rightarrow \mathrm{E} \mathrm{IX}$ & $a b c d e f g$ & $a b c d e f x z$ & $\begin{aligned} x= & -(a+2 c+3 d+2 f) \\
& -\frac{1}{2}(3 b+5 e+g) \\
z= & x-g\end{aligned}$ \\
\hline
\end{tabular}

Assume first that $\mathfrak{g}_{2}$ is of type EVI. Then the transformed labels, i.e., the labels of

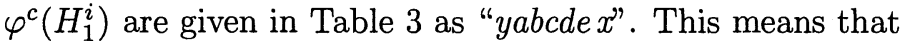

$$
\begin{aligned}
& \beta_{1}^{(2)}\left(\varphi^{c} H_{1}^{i}\right)=y, \quad \beta_{2}^{(2)}\left(\varphi^{c} H_{1}^{i}\right)=a, \quad \beta_{3}^{(2)}\left(\varphi^{c} H_{1}^{i}\right)=b, \quad \beta_{4}^{(2)}\left(\varphi^{c} H_{1}^{i}\right)=c, \\
& \beta_{5}^{(2)}\left(\varphi^{c} H_{1}^{i}\right)=d, \quad \beta_{6}^{(2)}\left(\varphi^{c} H_{1}^{i}\right)=e, \quad \beta_{7}^{(2)}\left(\varphi^{c} H_{1}^{i}\right)=x .
\end{aligned}
$$

This is illustrated on Figure 2. These data are in agreement with Table 1 which says that $\varphi^{c}$ maps the root spaces of $\mathfrak{g}_{1}^{c}$ corresponding to the roots $\beta_{k}^{(1)}$ to those of $\mathfrak{g}_{2}^{c}$ corresponding to the roots $\beta_{k+1}^{(2)}$ for $1 \leq k \leq 5$, and the root space of $\beta_{6}^{(1)}=\alpha_{6}^{(1)}$ to that of $\alpha_{6}^{(2)}$. We still need to compute the labels $\beta_{1}^{(2)}\left(\varphi^{c} H_{1}^{i}\right)=y$ and $\beta_{7}^{(2)}\left(\varphi^{c} H_{1}^{i}\right)=x$. Observe that $\varphi^{c}\left(\mathfrak{h}_{1}^{c}\right)$ is precisely the kernel of the fundamental weight $\omega_{7}^{(2)}$. As

$$
2 \omega_{7}^{(2)}=2 \alpha_{1}^{(2)}+3 \alpha_{2}^{(2)}+4 \alpha_{3}^{(2)}+6 \alpha_{4}^{(2)}+5 \alpha_{5}^{(2)}+4 \alpha_{6}^{(2)}+3 \alpha_{7}^{(2)},
$$

we obtain the equation

$$
2 a+3 e+4 b+6 c+5 d+4 f+3 x=0 .
$$

Since $\tilde{\alpha}^{(2)}$ is the highest root of $\tilde{R}_{2}$, we also have

$$
x+y+2(a+e+f)+3(b+d)+4 c=0 .
$$

From these two equations we obtain the formulae for $x$ and $y$ given in Table 3 . 


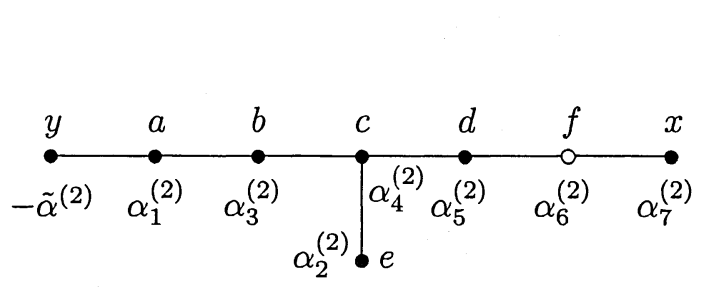

$$
\mathrm{E} \text { III } \rightarrow \mathrm{E} \mathrm{VI}
$$

$$
\alpha_{k}^{(1)} \rightarrow \alpha_{k}^{(2)} \quad(1 \leq k \leq 6)
$$

abcde $f$

yabcde $x$

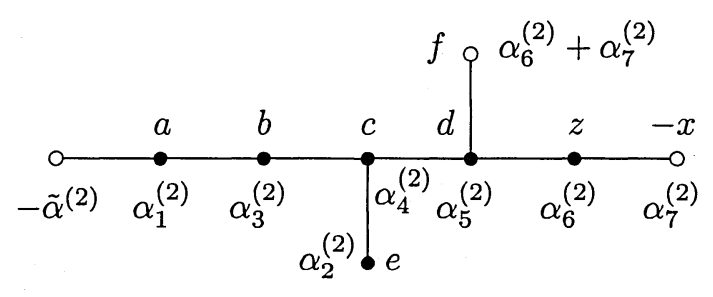

$\mathrm{E} \mathrm{III} \rightarrow$ E VII

$$
\begin{aligned}
& \alpha_{k}^{(1)} \rightarrow \alpha_{k}^{(2)} \quad(1 \leq k \leq 5) \\
& \alpha_{6}^{(1)} \rightarrow \alpha_{6}^{(2)}+\alpha_{7}^{(2)} \\
& \begin{array}{ll}
\text { abcde } & f \\
\text { aebcdz } & -x
\end{array}
\end{aligned}
$$

Figure 2. Two $\mathbf{Z}_{2}$-embeddings of inner type

Next assume that $\mathfrak{g}_{2}$ is of type E VII. Then the labels of $\varphi^{c}\left(H_{1}^{i}\right)$ are given in Table 3 as "aebcdz-x". This means that

$$
\begin{array}{ll}
\beta_{1}^{(2)}\left(\varphi^{c} H_{1}^{i}\right)=a, & \beta_{2}^{(2)}\left(\varphi^{c} H_{1}^{i}\right)=e, \quad \beta_{3}^{(2)}\left(\varphi^{c} H_{1}^{i}\right)=b, \quad \beta_{4}^{(2)}\left(\varphi^{c} H_{1}^{i}\right)=c \\
\beta_{5}^{(2)}\left(\varphi^{c} H_{1}^{i}\right)=d, \quad \beta_{6}^{(2)}\left(\varphi^{c} H_{1}^{i}\right)=z, \quad \beta_{7}^{(2)}\left(\varphi^{c} H_{1}^{i}\right)=-x
\end{array}
$$

and that the root spaces of $\mathfrak{g}_{1}^{c}$ corresponding to $\beta_{1}^{(2)}, \beta_{2}^{(2)}, \beta_{3}^{(2)}, \beta_{4}^{(2)}, \beta_{5}^{(2)}$ are mapped to those of $\mathfrak{g}_{2}^{c}$ corresponding to $\alpha_{1}^{(2)}, \alpha_{3}^{(2)}, \alpha_{4}^{(2)}, \alpha_{5}^{(2)}, \alpha_{2}^{(2)}$, respectively. As indicated in Table 1 (see also Figure 2), the root space of $\beta_{6}^{(1)}$ is mapped to that of $\alpha_{6}^{(2)}+\alpha_{7}^{(2)}$. Consequently, $f=z-x$.

In this case, $\varphi^{c}\left(\mathfrak{h}_{1}^{c}\right)$ is the kernel of $\omega_{6}^{(2)}-\omega_{7}^{(2)}$. As

$$
2\left(\omega_{6}^{(2)}-\omega_{7}^{(2)}\right)=2 \alpha_{1}^{(2)}+3 \alpha_{2}^{(2)}+4 \alpha_{3}^{(2)}+6 \alpha_{4}^{(2)}+5 \alpha_{5}^{(2)}+4 \alpha_{6}^{(2)}+\alpha_{7}^{(2)},
$$

we obtain the equation

$$
2 a+3 e+4 b+6 c+5 d+4 z-x=0 .
$$

As $z=f+x$, we obtain the same formula for $x$ as in the previous case.

REMARK 2.4. It follows from the transformation rules given in Table 3 that the labels given in column 2 of this table satisfy the following arithmetic conditions:

(i) $c \equiv d(\bmod 2) \quad$ if $\mathfrak{g}$ is of type $F I$,

(ii) $a \equiv c(\bmod 2) \quad$ if $\mathfrak{g}$ is of type $F I I$, 
(iii) $a+2 b+3 c \equiv e+2 d+3 f(\bmod 6)$ if $\mathfrak{g}$ is of type $E I I$,

(iv) $a+d \equiv b+f(\bmod 3)$ if $\mathfrak{g}$ is of type EIII,

(v) $a+2 b+e \equiv c+2 f+g(\bmod 4)$ if $\mathfrak{g}$ is of type $E V$, and

(vi) $e+f \equiv g(\bmod 2) \quad$ if $\mathfrak{g}$ is of type $E V I$.

Of course they can be easily verified by inspecting the tables given in the Appendix.

3. Embeddings from the diagram (1.3). We consider first the $\mathbf{Z}_{2}$-embedding $\mathfrak{g}_{1} \rightarrow \mathfrak{g}_{2}$ where $\mathfrak{g}_{1}$ is of type $\mathfrak{s o}(5,4)$ and $\mathfrak{g}_{2}$ of type FI. The nilpotent $G_{1}^{c}$-orbits in $\mathfrak{g}_{1}^{c}$ are parametrized by the partitions of 9 in which the even parts occur in pairs. They are listed in the first column of Table 5. The second and third columns give the $a b$-diagram and the right superscript (when needed) which parametrize the $K_{1}^{c}$-orbits in $\mathfrak{p}_{1}^{c}$. For more details about this notation we refer the reader to [9]. We warn the reader that the group $K$ in that paper is disconnected, but the orbits of its identity component are the same as the orbits of the group $K_{1}^{c}$ of this paper.

TABLE 4. The simple roots of $\tilde{R}$ and $R$

$\mathfrak{g} \quad \mathfrak{k}^{c} \quad \tilde{R}$ and $R$

$\mathfrak{s o}(4,3)$

$2 A_{1}+\tilde{A}_{1}$

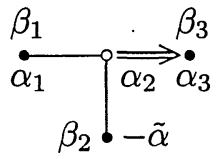

$\mathfrak{s o}(4,4)$

$4 A_{1}$

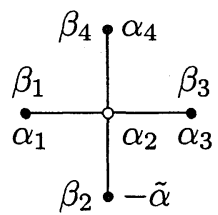

$\mathfrak{s o}(5,4)$

$B_{2}+2 A_{1}$

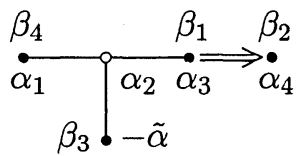

In the fourth column of Table 5 we assign a number $i$ to each of the nonzero nilpotent $K_{1}^{c}$-orbits in $\mathfrak{p}_{1}^{c}$, and in the fifth column we list the labels of the characteristics $H^{i}$ of these orbits. These labels are written as " $a b c d$ " where $a=\beta_{1}\left(H^{i}\right), b=\beta_{2}\left(H^{i}\right)$, $c=\beta_{3}\left(H^{i}\right), d=\beta_{4}\left(H^{i}\right)$. See the last diagram in Table 4 for the definition of these $\beta_{j}$ 's.

The number in the last column indicates the nilpotent $K_{2}^{c}$-orbit (see Table 10 for the enumeration of these orbits) that contains the image of the given $K_{1}^{c}$-orbit. These numbers are computed by using the same technique as in the previous section. The transformation rule in this case is " $a b c d$ " $\rightarrow$ " $x b a d$ " where $x=-b+\frac{1}{2}(c-a)$. 
TABLE 5. Nonzero nilpotent $K^{c}$-orbits in $\mathfrak{p}^{c}$ for $\mathfrak{g}=\mathfrak{s o}(5,4)$

\begin{tabular}{|l|ll|r|r|r|}
\hline Partition & $a b$-diagram & r.s. & No. & Labels & F I \\
\hline $1^{5} 2^{2}$ & $a b, b a, a^{3}, b^{2}$ & & 1 & 1011 & 1 \\
\hline $1^{6} 3$ & $b a b, a^{4}, b^{2}$ & & 2 & 0022 & 2 \\
& $a b a, a^{3}, b^{3}$ & & 3 & 2000 & 3 \\
\hline $1 \cdot 2^{4}$ & $(a b, b a)^{2}, a$ & I & 4 & 0102 & 2 \\
& & II & 5 & 0120 & 3 \\
\hline $1^{2} 2^{2} 3$ & $b a b, a b, b a, a^{2}$ & I & 6 & 1013 & 4 \\
& & II & 7 & 1031 & 5 \\
& $a b a, a b, b a, a, b$ & & 8 & 1111 & 5 \\
\hline $1^{3} 3^{2}$ & $(b a b)^{2}, a^{3}$ & I & 9 & 0004 & 6 \\
& & II & 10 & 0040 & 7 \\
& $a b a, b a b, a^{2}, b$ & & 11 & 2022 & 8 \\
& $(a b a)^{2}, a, b^{2}$ & & 12 & 0200 & 7 \\
\hline $3^{3}$ & $(a b a)^{2}, b a b$ & & 13 & 0222 & 10 \\
\hline $1^{4} 5$ & $(b a)^{2} b, a^{3}, b$ & & 14 & 2044 & 11 \\
& $(a b)^{2} a, a^{2}, b^{2}$ & & 15 & 4022 & 12 \\
\hline $1 \cdot 4^{2}$ & $(a b)^{2},(b a)^{2}, a$ & I & 16 & 2124 & 11 \\
& & II & 17 & 2142 & 12 \\
\hline $2^{2} 5$ & $(a b)^{2} a, a b, b a$ & I & 18 & 3113 & 15 \\
& & II & 19 & 3131 & 14 \\
\hline $1 \cdot 3 \cdot 5$ & $(b a)^{2} b, a b a, a$ & & 20 & 0244 & 17 \\
& $(a b)^{2} a, b a b, a$ & I & 21 & 4004 & 17 \\
& & II & 22 & 4040 & 16 \\
& $(a b)^{2} a, a b a, b$ & & 23 & 2222 & 18 \\
\hline $1^{2} 7$ & $(b a)^{3} b, a^{2}$ & I & 24 & 4048 & 19 \\
& & II & 25 & 4084 & 20 \\
& $(a b)^{3} a, a, b$ & & 26 & 4244 & 20 \\
\hline 9 & $(a b)^{4} a$ & 27 & 4448 & 25 \\
& & II & 28 & 4484 & 24 \\
\hline
\end{tabular}

Next we consider the $\mathbf{Z}_{2}$-embedding $\mathfrak{g}_{1} \rightarrow \mathfrak{g}_{2}$ where $\mathfrak{g}_{1}$ is of type $\mathfrak{s o}(4,4)$ and $\mathfrak{g}_{2}$ of type $\mathfrak{s o}(5,4)$. The nilpotent $G_{1}^{c}$-orbits in $\mathfrak{g}_{1}^{c}$ are parametrized by the partitions of 8 in which the even parts occur in pairs, except that to each of the two very even partitions $\left(2^{4}\right.$ and $\left.4^{2}\right)$ there correspond two orbits. These partitions are listed in the first column of Table 6 . The next three columns give the $a b$-diagrams and the left and/or right superscripts (when needed) which parametrize the $K_{1}^{c}$-orbits in $\mathfrak{p}_{1}^{c}$ (see [9] for details).

In the fifth column of Table 6 we assign a number $i$ to each of the nonzero nilpotent $K_{1}^{c}$-orbits in $\mathfrak{p}_{1}^{c}$, and in the sixth column we list the labels $\beta_{j}\left(H^{i}\right), 1 \leq j \leq 4$, of the characteristics $H^{i}$ (see Table 4 for the definition of the $\beta_{j}$ 's). 
TABLE 6. Nonzero nilpotent $K^{c}$-orbits in $\mathfrak{p}^{c}$ for $\mathfrak{g}=\mathfrak{s o}(4,4)$

\begin{tabular}{|c|c|c|c|c|c|c|}
\hline Partition & l.s. & $a b$-diagram & r.s. & No. & Labels & $\mathfrak{s o}(5,4)$ \\
\hline $1^{4} 2^{2}$ & & $a b, b a, a^{2}, b^{2}$ & & 1 & 1111 & 1 \\
\hline \multirow[t]{2}{*}{$1^{5} 3$} & & $a b a, a^{2}, b^{3}$ & & 2 & 2200 & 3 \\
\hline & & $b a b, a^{3}, b^{2}$ & & 3 & 0022 & 2 \\
\hline \multirow[t]{4}{*}{$2^{4}$} & I & $(a b, b a)^{2}$ & $\bar{I}$ & 4 & 0202 & 4 \\
\hline & I & & II & 5 & 0220 & 5 \\
\hline & II & & I & 6 & 2002 & 4 \\
\hline & II & & II & 7 & 2020 & 5 \\
\hline \multirow[t]{4}{*}{$1 \cdot 2^{2} 3$} & I & $a b a, a b, b a, b$ & & 8 & 1311 & 8 \\
\hline & II & & & 9 & 3111 & 8 \\
\hline & & $b a b, a b, b a, a$ & I & 10 & 1113 & 6 \\
\hline & & & II & 11 & 1131 & 7 \\
\hline \multirow[t]{5}{*}{$1^{2} 3^{2}$} & I & $(a b a)^{2}, b^{2}$ & & 12 & 0400 & 12 \\
\hline & II & & & 13 & 4000 & 12 \\
\hline & & $a b a, b a b, a, b$ & & 14 & 2222 & 11 \\
\hline & & $(b a b)^{2}, a^{2}$ & I & 15 & 0004 & 9 \\
\hline & & & II & 16 & 0040 & 10 \\
\hline \multirow[t]{2}{*}{$1^{3} 5$} & & $(a b)^{2} a, a, b^{2}$ & & 17 & 4422 & 15 \\
\hline & & $(b a)^{2} b, a^{2}, b$ & & 18 & 2244 & 14 \\
\hline \multirow[t]{4}{*}{$4^{2}$} & I & $(a b)^{2},(b a)^{2}$ & $\mathrm{I}$ & 19 & 2424 & 16 \\
\hline & I & & II & 20 & 2442 & 17 \\
\hline & II & & I & 21 & 4224 & 16 \\
\hline & II & & II & 22 & 4242 & 17 \\
\hline \multirow[t]{4}{*}{$3 \cdot 5$} & & $(a b)^{2} a, b a b$ & I & 23 & 4404 & 21 \\
\hline & & & II & 24 & 4440 & 22 \\
\hline & I & $(b a)^{2} b, a b a$ & & 25 & 0444 & 20 \\
\hline & II & & & 26 & 4044 & 20 \\
\hline \multirow[t]{4}{*}{$1 \cdot 7$} & I & $(a b)^{3} a, b$ & & 27 & 4844 & 26 \\
\hline & II & & & 28 & 8444 & 26 \\
\hline & & $(b a)^{3} b, a$ & I & 29 & 4448 & 24 \\
\hline & & & II & 30 & 4484 & 25 \\
\hline
\end{tabular}

The last column gives the number (from Table 5) of the nilpotent $K_{2}^{c}$-orbit that contains the given $K_{1}^{c}$-orbit. They were computed by using the transformation rule " $a b c d " \rightarrow$ "ax $c d$ " where $x=\frac{1}{2}(b-a)$.

Next we consider $\mathfrak{g}_{1} \rightarrow \mathfrak{g}_{2}$ where $\mathfrak{g}_{1}$ is of type $\mathfrak{s o}(4,3)$ and $\mathfrak{g}_{2}$ of type $\mathfrak{s o}(4,4)$. The nilpotent $G_{1}^{c}$-orbits in $\mathfrak{g}_{1}^{c}$ are parametrized by the partitions of 7 in which the even parts occur in pairs. These partitions are listed in the first column of Table 7 . The next two columns give the $a b$-diagrams and the left superscripts (when needed) which parametrize the $K_{1}^{c}$-orbits in $\mathfrak{p}_{1}^{c}$.

In the fourth column we assign a number $i$ to each of the nonzero nilpotent $K_{1}^{c}$ orbits in $\mathfrak{p}_{1}^{c}$, and in the fifth column we list the labels $\beta_{j}\left(H^{i}\right), 1 \leq j \leq 3$, of the characteristics $H^{i}$ (see Table 4). 
TABLE 7. Nonzero nilpotent $K^{c}$-orbits in $\mathfrak{p}^{c}$ for $\mathfrak{g}=\mathfrak{s o}(4,3)$

\begin{tabular}{|l|ll|r|l|r|}
\hline Partition & l.s. & $a b$-diagram & No. & Labels & so(4,4) \\
\hline $1^{3} 2^{2}$ & & $a b, b a, a^{2}, b$ & 1 & 111 & 1 \\
\hline $1^{4} 3$ & & $a b a, a^{2}, b^{2}$ & 2 & 220 & 2 \\
& & $b a b, a^{3}, b$ & 3 & 002 & 3 \\
\hline $2^{2} 3$ & I & $a b a, a b, b a$ & 4 & 131 & 8 \\
& II & & 5 & 311 & 9 \\
\hline $1 \cdot 3^{2}$ & I & $(a b a)^{2}, b$ & 6 & 040 & 12 \\
& II & & 7 & 400 & 13 \\
& & $a b a, b a b, a$ & 8 & 222 & 14 \\
\hline $1^{2} 5$ & & $(a b)^{2} a, a, b$ & 9 & 442 & 17 \\
& & $(b a)^{2} b, a^{2}$ & 10 & 224 & 18 \\
\hline 7 & I & $(a b)^{3} a$ & 11 & 484 & 27 \\
& II & & 12 & 844 & 28 \\
\hline
\end{tabular}

The last column gives the number (from Table 6) of the nilpotent $K_{2}^{c}$-orbit that contains the given $K_{1}^{c}$-orbit. The transformation rule in this case is " $a b c$ " $\rightarrow$ " $a b c c$ ".

There is a $\mathbf{Z}_{2}$-embedding $\mathfrak{g}_{1} \rightarrow \mathfrak{g}_{2}$ with $\mathfrak{g}_{1}$ of type GI and $\mathfrak{g}_{2}=\mathfrak{s o}(4,3)$, with the transformation rule " $a b$ " $\rightarrow$ " $a b a$ ". Under this embedding the nonzero nilpotent $K_{1}^{c}$-orbits in $\mathfrak{p}_{1}^{c}$ are mapped to those of $K_{2}^{c}$ in $\mathfrak{p}_{2}^{c}$ as follows:

$$
1 \rightarrow 1, \quad 2 \rightarrow 4, \quad 3 \rightarrow 8, \quad 4 \rightarrow 6, \quad 5 \rightarrow 11 .
$$

Finally for the $\mathbf{Z}_{2}$-embedding of $\mathfrak{s l}(3, \mathbf{R})$ into the algebra $\mathfrak{g}_{2}$ of type $G I$, the minimal nonzero nilpotent $K_{1}^{c}$-orbit in $\mathfrak{p}_{1}^{c}$ is mapped into the orbit 1 and the principal orbit is mapped into the orbit 3.

4. Appendix: Enumeration of the $K^{c}$-orbits in $\mathfrak{p}^{c}$. For the reader's convenience, we give here the parametrization of the nonzero nilpotent $K^{c}$-orbits in $\mathfrak{p}^{c}$ for $\mathfrak{g}$ of exceptional type, which is taken from $[5,6]$ but is presented here in a different form (using Bala-Carter symbols). For the sake of consistency, we use the same numbering of orbits as in these two papers. They are also listed in [4].

For each of the real forms $\mathfrak{g}$ we give in Table 8 the Dynkin diagram of the root system $R$ of $\left(\mathfrak{k}^{c}, \mathfrak{h}^{c}\right)$. The nodes of this Dynkin diagram are the black nodes. They are labeled by the simple roots $\beta_{1}, \beta_{2}, \ldots$.

If $\mathfrak{g}$ is of inner type, then this diagram is embedded in the extended Dynkin diagram of the root system $\tilde{R}$ of $\left(\mathfrak{g}^{c}, \mathfrak{h}^{c}\right)$. The simple roots of $\tilde{R}$ are denoted by $\alpha_{1}, \alpha_{2}, \ldots$, and its highest root by $\tilde{\alpha}$. If to the simple roots of $\tilde{R}$ we assign the weights: 0 for black nodes and 1 for the single white node, then we obtain a $\mathbf{Z}$-gradation of $\mathfrak{g}^{c}$ whose associated $\mathbf{Z}_{2}$-gradation is $\mathfrak{g}^{c}=\mathfrak{k}^{c} \oplus \mathfrak{p}^{c}$.

If $\mathfrak{g}$ is of type EIII or EVII, then $\mathfrak{k}^{c}$ is not semisimple and we need another root from $\tilde{R}$ to specify the characteristics $H \in \mathfrak{h}^{c}$. For that purpose we use the root $\beta_{6}$ for EIII and $\beta_{7}$ for E VII. Note that both of these nodes are white. 
TABLE 8. The simple roots of $\tilde{R}$ and $R$

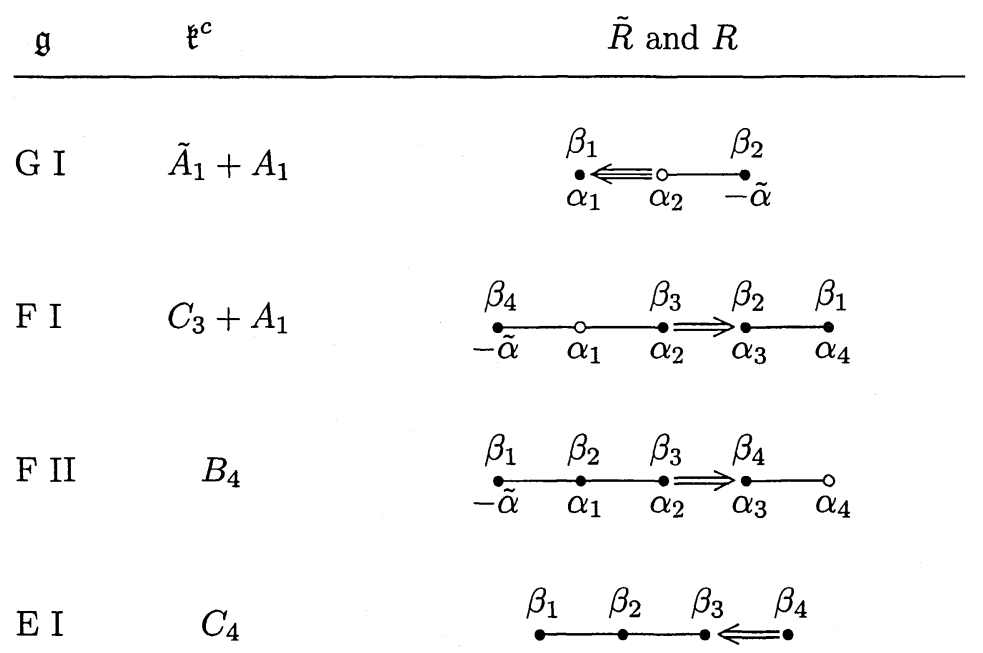

E II $\quad A_{5}+A_{1}$

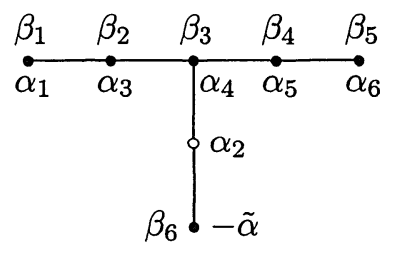

E III $\quad D_{5}+T_{1}$

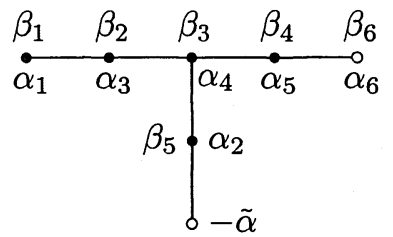

E IV

$F_{4}$

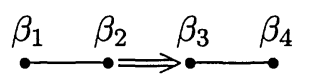

E V

$$
A_{7}
$$

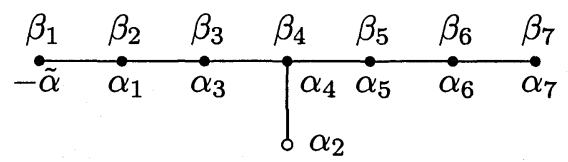




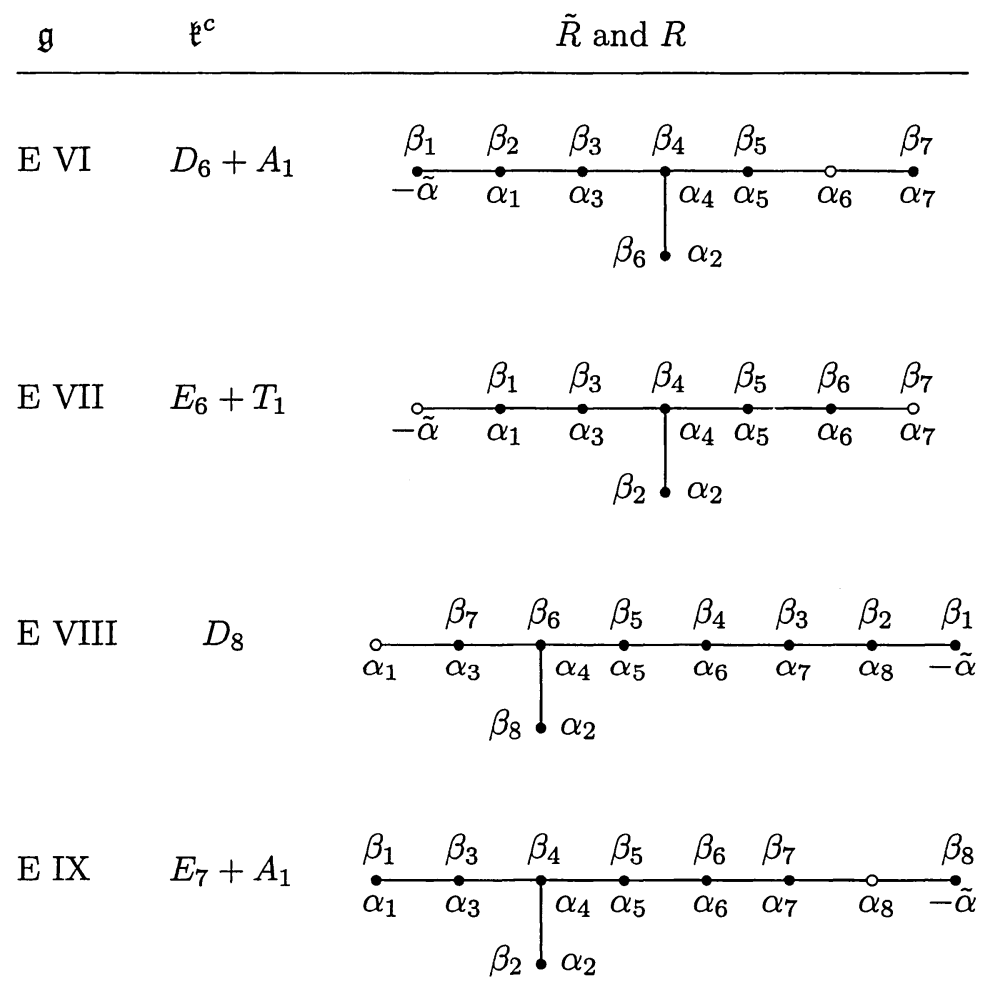

If $H^{i}$ is the characteristic of the $i$-th orbit, then the labels $\beta_{j}\left(H^{i}\right)$ determine $H^{i}$ uniquely. All these labels are nonnegative integers except the one corresponding to the white $\beta$ node which may be a negative integer.

There is only one misprint in the list of characteristics in $[5,6]$, namely the one for the orbit 31 of [5, Table XII]. These labels should be 0202202 instead of 0202200 . This misprint was also copied into [4, p. 158]. We warn the reader that the Dynkin diagram of $F_{4}$ in [4, p. 152] should have its direction arrow reversed.

The B-C columns in Tables 9-20 of this appendix give the Bala-Carter symbols for nonzero nilpotent $G^{c}$-orbits $\mathcal{O}$ in $\mathfrak{g}^{c}$. As in the introduction, let $\mathcal{B}_{1}, \ldots, \mathcal{B}_{k}$ be the connected components of $\mathcal{O} \cap \mathfrak{p}^{c}$. Each of these components is given a number, say $i$, which is followed by the labels $\beta_{j}\left(H^{i}\right)$ of the characteristic $H^{i}$ of that component.

For instance if $\mathfrak{g}$ is of type EII and $\mathcal{O}$ has the Bala-Carter symbol $E_{6}\left(a_{3}\right)$, then $k=2$ and the two connected components are given the numbers 32 and 33 (see Table 13). Since $\mathfrak{k}^{c}$ is of type $A_{5}+A_{1}$, we have separated the first five labels (corresponding to $A_{5}$ ) from the last one (corresponding to $A_{1}$ ) by a blank space. For the orbit 32 , all labels $\beta_{j}\left(H^{32}\right), 1 \leq j \leq 6$, are equal to 2. (See the diagram EII in Table 8 for the definition of the roots $\beta_{j}$.) For the orbit 33 , the labels $\beta_{j}\left(H^{33}\right)$ are 0 for $j$ odd and 4 for $j$ even. 
TABLE 9. Nonzero nilpotent $K^{c}$-orbits in $\mathfrak{p}^{c}$ for $\mathfrak{g}$ of type $G I$

\begin{tabular}{|lrl|lrl|}
\hline B-C symbol & No. & Labels & B-C symbol & No. & Labels \\
\hline$A_{1}$ & 1 & 11 & $G_{2}\left(a_{1}\right)$ & 4 & 04 \\
$\tilde{A}_{1}$ & 2 & 13 & $G_{2}$ & 5 & 48 \\
$G_{2}\left(a_{1}\right)$ & 3 & 22 & & & \\
\hline
\end{tabular}

TABLE 10. Nonzero nilpotent $K^{c}$-orbits in $\mathfrak{p}^{c}$ for $\mathfrak{g}$ of type $F I$

\begin{tabular}{|lrl|lrl|}
\hline B-C symbol & No. & Labels & B-C symbol & No. & Labels \\
\hline$A_{1}$ & 1 & 0011 & $C_{3}\left(a_{1}\right)$ & 14 & 1031 \\
$\tilde{A}_{1}$ & 2 & 1002 & & 15 & 1113 \\
& 3 & 0100 & $F_{4}\left(a_{3}\right)$ & 16 & 0040 \\
$A_{1}+\tilde{A}_{1}$ & 4 & 0013 & & 17 & 0204 \\
& 5 & 1011 & & 18 & 2022 \\
$A_{2}$ & 6 & 0004 & $B_{3}$ & 19 & 0048 \\
& 7 & 2000 & & 20 & 2044 \\
& 8 & 0022 & $C_{3}$ & 21 & 1313 \\
$\tilde{A}_{2}$ & 9 & 0200 & $F_{4}\left(a_{2}\right)$ & 22 & 0404 \\
$A_{2}+\tilde{A}_{1}$ & 10 & 1102 & & 23 & 2222 \\
$B_{2}$ & 11 & 1024 & $F_{4}\left(a_{1}\right)$ & 24 & 2244 \\
& 12 & 0122 & & 25 & 4048 \\
$\tilde{A}_{2}+A_{1}$ & 13 & 1111 & $F_{4}$ & 26 & 4448 \\
\hline
\end{tabular}

TABLE 11. Nonzero nilpotent $K^{c}$-orbits in $\mathfrak{p}^{c}$ for $\mathfrak{g}$ of type $F I I$

\begin{tabular}{|lrl|}
\hline B-C symbol & No. & Labels \\
\hline$\tilde{A}_{1}$ & 1 & 0001 \\
$\tilde{A}_{2}$ & 2 & 4000 \\
\hline
\end{tabular}

TABLE 12. Nonzero nilpotent $K^{c}$-orbits in $\mathfrak{p}^{c}$ for $\mathfrak{g}$ of type $E I$

\begin{tabular}{|lrl|lrl|}
\hline B-C symbol & No. & Labels & B-C symbol & No. & Labels \\
\hline$A_{1}$ & 1 & 0001 & $D_{4}\left(a_{1}\right)$ & 23 & 0020 \\
$2 A_{1}$ & 2 & 0100 & $A_{4}$ & 9 & 0202 \\
$3 A_{1}$ & 3 & 1001 & $D_{4}$ & 13 & 2004 \\
$A_{2}$ & 4 & 0002 & $A_{4}+A_{1}$ & 16 & 1111 \\
& 5 & 2000 & $D_{5}\left(a_{1}\right)$ & 17 & 1112 \\
$A_{2}+A_{1}$ & 8 & 0101 & $A_{5}$ & 14 & 1211 \\
$2 A_{2}$ & 6 & 0200 & $E_{6}\left(a_{3}\right)$ & 19 & 2202 \\
$A_{2}+2 A_{1}$ & 10 & 1010 & & 22 & 0220 \\
$A_{3}$ & 7 & 0102 & $D_{5}$ & 21 & 2204 \\
$2 A_{2}+A_{1}$ & 11 & 1101 & $E_{6}\left(a_{1}\right)$ & 18 & 2222 \\
$A_{3}+A_{1}$ & 15 & 1011 & $E_{6}$ & 20 & 4224 \\
$D_{4}\left(a_{1}\right)$ & 12 & 2002 & & & \\
\hline
\end{tabular}


TABLE 13. Nonzero nilpotent $K^{c}$-orbits in $\mathfrak{p}^{c}$ for $\mathfrak{g}$ of type EII

\begin{tabular}{|lrl|lrl|}
\hline B-C symbol & No. & Labels & B-C symbol & No. & Labels \\
\hline$A_{1}$ & 1 & 001001 & $D_{4}\left(a_{1}\right)$ & 20 & 004000 \\
$2 A_{1}$ & 2 & 100012 & & 21 & 020204 \\
& 3 & 010100 & & 22 & 202022 \\
$3 A_{1}$ & 4 & 001003 & $A_{4}$ & 25 & 400044 \\
& 5 & 101011 & & 26 & 220220 \\
$A_{2}$ & 6 & 000004 & $D_{4}$ & 23 & 004008 \\
& 7 & 200020 & & 24 & 204024 \\
& 8 & 002002 & $A_{4}+A_{1}$ & 27 & 121131 \\
$A_{2}+A_{1}$ & 9 & 210011 & & 28 & 311211 \\
& 10 & 100121 & $D_{5}\left(a_{1}\right)$ & 29 & 313104 \\
$2 A_{2}$ & 11 & 020200 & & 30 & 013134 \\
$A_{2}+2 A_{1}$ & 12 & 301000 & $A_{5}$ & 31 & 131313 \\
& 13 & 001030 & $E_{6}\left(a_{3}\right)$ & 32 & 222222 \\
& 14 & 110112 & & 33 & 040404 \\
$A_{3}$ & 15 & 102014 & $D_{5}$ & 34 & 224224 \\
& 16 & 012102 & & 35 & 404048 \\
$2 A_{2}+A_{1}$ & 17 & 111111 & $E_{6}\left(a_{1}\right)$ & 36 & 440444 \\
$A_{3}+A_{1}$ & 18 & 103011 & $E_{6}$ & 37 & 444448 \\
& 19 & 111113 & & & \\
\hline
\end{tabular}

TABLE 14. Nonzero nilpotent $K^{c}$-orbits in $\mathfrak{p}^{c}$ for $\mathfrak{g}$ of type EIII

\begin{tabular}{|lrlr|lrll|}
\hline B-C symbol & No. & Labels & & B-C symbol & No. & Labels & \\
\hline$A_{1}$ & 1 & 00001 & 0 & $A_{2}+A_{1}$ & 7 & 11010 & -2 \\
& 2 & 00010 & -2 & & 8 & 11001 & -3 \\
$2 A_{1}$ & 3 & 10000 & 1 & $2 A_{2}$ & 9 & 40000 & -2 \\
& 4 & 10000 & -2 & $A_{3}$ & 10 & 00013 & -2 \\
& 5 & 00011 & -2 & & 11 & 00031 & -6 \\
$A_{2}$ & 6 & 02000 & -2 & $A_{4}$ & 12 & 02022 & -6 \\
\hline
\end{tabular}

TABLE 15. Nonzero nilpotent $K^{c}$-orbits in $\mathfrak{p}^{c}$ for $\mathfrak{g}$ of type EIV

\begin{tabular}{|lrl|}
\hline B-C symbol & No. & Labels \\
\hline$A_{1}$ & 1 & 0001 \\
$2 A_{1}$ & 2 & 0002 \\
\hline
\end{tabular}


TABLE 16. Nonzero nilpotent $K^{c}$-orbits in $\mathfrak{p}^{c}$ for $\mathfrak{g}$ of type $E V$

\begin{tabular}{|c|c|c|c|c|c|}
\hline B-C symbol & No. & Labels & B-C symbol & No. & Labels \\
\hline$\overline{A_{1}}$ & 1 & 0001000 & $A_{4}+A_{1}$ & 48 & 3101021 \\
\hline $2 A_{1}$ & 2 & 0100010 & & 49 & 1201013 \\
\hline \multirow[t]{2}{*}{$\left(3 A_{1}\right)^{\prime \prime}$} & 3 & 0200000 & & 50 & 1111111 \\
\hline & 4 & 0000020 & $D_{5}\left(a_{1}\right)$ & 51 & 3013010 \\
\hline$\left(3 A_{1}\right)^{\prime}$ & 5 & 1001001 & & 52 & 0103103 \\
\hline \multirow[t]{2}{*}{$A_{2}$} & 6 & 2000002 & & 53 & 1112111 \\
\hline & 7 & 0002000 & $A_{4}+A_{2}$ & 54 & 2020202 \\
\hline \multirow[t]{2}{*}{$4 A_{1}$} & 8 & 1100100 & $D_{5}\left(a_{1}\right)+A_{1}$ & 55 & 4004000 \\
\hline & 9 & 0010011 & & 56 & 0004004 \\
\hline \multirow[t]{3}{*}{$A_{2}+A_{1}$} & 10 & 2010001 & & 57 & 2022020 \\
\hline & 11 & 1000102 & & 58 & 0202202 \\
\hline & 12 & 0101010 & $\left(A_{5}\right)^{\prime}$ & 59 & 1211121 \\
\hline \multirow[t]{3}{*}{$A_{2}+2 A_{1}$} & 13 & 3000100 & $A_{5}+A_{1}$ & 60 & 1311111 \\
\hline & 14 & 0010003 & & 61 & 1111131 \\
\hline & 15 & 1010101 & $E_{6}\left(a_{3}\right)$ & 62 & 2202022 \\
\hline \multirow[t]{4}{*}{$A_{2}+3 A_{1}$} & 16 & 4000000 & & 63 & 0220220 \\
\hline & 17 & 0000004 & $D_{6}\left(a_{2}\right)$ & 64 & 1310301 \\
\hline & 18 & 2000200 & & 65 & 1030131 \\
\hline & 19 & 0020002 & $D_{5}$ & 66 & 2204022 \\
\hline$A_{3}$ & 20 & 0102010 & $E_{7}\left(a_{5}\right)$ & 67 & 2220202 \\
\hline $2 A_{2}$ & 21 & 0200020 & & 68 & 2020222 \\
\hline \multirow[t]{2}{*}{$\left(A_{3}+A_{1}\right)^{\prime \prime}$} & 22 & 0202000 & & 69 & 0400400 \\
\hline & 23 & 0002020 & & 70 & 0040040 \\
\hline $2 A_{2}+A_{1}$ & 24 & 1101011 & $A_{6}$ & 71 & 2220222 \\
\hline$\left(A_{3}+A_{1}\right)^{\prime}$ & 25 & 1011101 & $D_{6}\left(a_{1}\right)$ & 72 & 3013131 \\
\hline \multirow[t]{2}{*}{$D_{4}\left(a_{1}\right)$} & 26 & 2002002 & & 73 & 1313103 \\
\hline & 27 & 0020200 & $D_{5}+A_{1}$ & 74 & 3113121 \\
\hline \multirow[t]{2}{*}{$A_{3}+2 A_{1}$} & 28 & 1111010 & & 75 & 1213113 \\
\hline & 29 & 0101111 & $E_{7}\left(a_{4}\right)$ & 76 & 2222202 \\
\hline$D_{4}$ & 30 & 2004002 & & 77 & 2022222 \\
\hline \multirow[t]{4}{*}{$D_{4}\left(a_{1}\right)+A_{1}$} & 31 & 2101101 & & 78 & 4004040 \\
\hline & 32 & 1011012 & & 79 & 0404004 \\
\hline & 33 & 0120101 & $E_{6}\left(a_{1}\right)$ & 80 & 4220224 \\
\hline & 34 & 1010210 & & 81 & 2222222 \\
\hline \multirow[t]{3}{*}{$A_{3}+A_{2}$} & 35 & 1030010 & $D_{6}$ & 82 & 3413131 \\
\hline & 36 & 0100301 & & 83 & 1313143 \\
\hline & 37 & 1110111 & $E_{6}$ & 84 & 4224224 \\
\hline \multirow[t]{2}{*}{$A_{4}$} & 38 & 2200022 & $E_{7}\left(a_{3}\right)$ & 85 & 2422222 \\
\hline & 43 & 0202020 & & 86 & 2222242 \\
\hline \multirow[t]{4}{*}{$A_{3}+A_{2}+A_{1}$} & 39 & 0040000 & & 87 & 4404040 \\
\hline & 40 & 0000400 & & 88 & 0404044 \\
\hline & 41 & 2020020 & $E_{7}\left(a_{2}\right)$ & 89 & 4404404 \\
\hline & 42 & 0200202 & & 90 & 4044044 \\
\hline \multirow[t]{2}{*}{$\left(A_{5}\right)^{\prime \prime}$} & 44 & 0402020 & $E_{7}\left(a_{1}\right)$ & 91 & 4444044 \\
\hline & 45 & 0202040 & & 92 & 4404444 \\
\hline
\end{tabular}


TABLE 16. (continued)

\begin{tabular}{|lrl|lrl|}
\hline B-C symbol & No. & Labels & B-C symbol & No. & Labels \\
\hline$D_{4}+A_{1}$ & 46 & 2103101 & $E_{7}$ & 93 & 8444444 \\
& 47 & 1013012 & & 94 & 4444448 \\
\hline
\end{tabular}

TABLE 17. Nonzero nilpotent $K^{c}$-orbits in $\mathfrak{p}^{c}$ for $\mathfrak{g}$ of type $E V I$

\begin{tabular}{|lrl|lrl|}
\hline B-C symbol & No. & Labels & B-C symbol & No. & Labels \\
\hline$A_{1}$ & 1 & 0000101 & $D_{4}\left(a_{1}\right)$ & 19 & 0000400 \\
$2 A_{1}$ & 2 & 0100002 & & 20 & 0002004 \\
& 3 & 0001000 & & 21 & 0200202 \\
$\left(3 A_{1}\right)^{\prime}$ & 4 & 0000103 & $D_{4}$ & 22 & 0000408 \\
& 5 & 0100101 & & 23 & 0200404 \\
$A_{2}$ & 6 & 0000004 & $A_{3}+A_{2}$ & 24 & 2010112 \\
& 7 & 0000202 & $A_{4}$ & 25 & 0400004 \\
& 8 & 0200000 & & 26 & 0202000 \\
$A_{2}+A_{1}$ & 9 & 1100011 & $A_{4}+A_{1}$ & 27 & 1111101 \\
$A_{2}+2 A_{1}$ & 10 & 2001000 & $D_{5}\left(a_{1}\right)$ & 28 & 2010314 \\
& 11 & 0101002 & $A_{4}+A_{2}$ & 29 & 0040000 \\
$A_{3}$ & 12 & 0100204 & $\left(A_{5}\right)^{\prime}$ & 30 & 0103103 \\
& 13 & 0001202 & $E_{6}\left(a_{3}\right)$ & 31 & 0202202 \\
$2 A_{2}$ & 14 & 4000000 & & 32 & 0004004 \\
& 15 & 0002000 & $D_{5}$ & 33 & 0202404 \\
$2 A_{2}+A_{1}$ & 16 & 0101101 & & 34 & 0400408 \\
$\left(A_{3}+A_{1}\right)^{\prime}$ & 17 & 0100301 & $A_{6}$ & 35 & 4004000 \\
& 18 & 0101103 & $E_{6}\left(a_{1}\right)$ & 36 & 0404004 \\
& & & $E_{6}$ & 37 & 0404408 \\
\hline
\end{tabular}

TABLE 18. Nonzero nilpotent $K^{c}$-orbits in $\mathfrak{p}^{c}$ for $\mathfrak{g}$ of type $E V I I$

\begin{tabular}{|lrlr|lrrr|}
\hline B-C symbol & No. & Labels & & B-C symbol & No. & Labels & \\
\hline$A_{1}$ & 1 & 100000 & 0 & $A_{2}+A_{1}$ & 12 & 011000 & -3 \\
& 2 & 000001 & -2 & $A_{3}$ & 13 & 300001 & -2 \\
$2 A_{1}$ & 3 & 000001 & 0 & & 14 & 100003 & -6 \\
& 4 & 100000 & -2 & $2 A_{2}$ & 15 & 200002 & -4 \\
$\left(3 A_{1}\right)^{\prime \prime}$ & 5 & 100001 & -2 & $\left(A_{3}+A_{1}\right)^{\prime \prime}$ & 16 & 200002 & -2 \\
& 6 & 000000 & 2 & & 17 & 400000 & -2 \\
& 7 & 000000 & -2 & & 18 & 000004 & -6 \\
& 8 & 000002 & -2 & & 19 & 200002 & -6 \\
$A_{2}$ & 9 & 200000 & -2 & $A_{4}$ & 20 & 220002 & -6 \\
$A_{2}+A_{1}$ & 10 & 020000 & -2 & $\left(A_{5}\right)^{\prime \prime}$ & 21 & 400004 & -6 \\
\hline
\end{tabular}


TABLE 19. Nonzero nilpotent $K^{c}$-orbits in $\mathfrak{p}^{c}$ for $\mathfrak{g}$ of type E VIII

\begin{tabular}{|c|c|c|c|c|c|}
\hline B-C symbol & No. & Labels & B-C symbol & No. & Labels \\
\hline$\overline{A_{1}}$ & 1 & 00000010 & $D_{5}\left(a_{1}\right)+A_{2}$ & 59 & 11010111 \\
\hline $2 A_{1}$ & 2 & 00010000 & $D_{6}\left(a_{2}\right)$ & 60 & 21011011 \\
\hline $3 A_{1}$ & 3 & 01000010 & & 61 & 10102100 \\
\hline \multirow[t]{2}{*}{$A_{2}$} & 4 & 02000000 & $E_{6}\left(a_{3}\right)+A_{1}$ & 62 & 11110110 \\
\hline & 5 & 00000020 & & 63 & 01011101 \\
\hline $4 A_{1}$ & 6 & 10001000 & $E_{7}\left(a_{5}\right)$ & 64 & 01003001 \\
\hline \multirow[t]{2}{*}{$A_{2}+A_{1}$} & 7 & 11000001 & & 65 & 11101101 \\
\hline & 8 & 00010010 & $D_{5}+A_{1}$ & 66 & 11110130 \\
\hline \multirow[t]{2}{*}{$A_{2}+2 A_{1}$} & 9 & 20010000 & $E_{8}\left(a_{7}\right)$ & 67 & 20200200 \\
\hline & 10 & 01000100 & & 68 & 00004000 \\
\hline$A_{3}$ & 11 & 00010020 & & 69 & 02002002 \\
\hline \multirow[t]{2}{*}{$A_{2}+3 A_{1}$} & 12 & 30000001 & $A_{6}$ & 70 & 40040000 \\
\hline & 13 & 10010001 & & 71 & 40040000 \\
\hline \multirow[t]{3}{*}{$2 A_{2}$} & 14 & 40000000 & $D_{6}\left(a_{1}\right)$ & 72 & 21011031 \\
\hline & 15 & 20000002 & & 73 & 01201031 \\
\hline & 16 & 00020000 & $A_{6}+A_{1}$ & 74 & 11111101 \\
\hline $2 A_{2}+A_{1}$ & 17 & 01010010 & $E_{7}\left(a_{4}\right)$ & 75 & 11101121 \\
\hline$A_{3}+A_{1}$ & 18 & 01000110 & & 76 & 10300130 \\
\hline \multirow[t]{2}{*}{$D_{4}\left(a_{1}\right)$} & 19 & 02000020 & $E_{6}\left(a_{1}\right)$ & 77 & 04020200 \\
\hline & 20 & 00000200 & & 78 & 02020220 \\
\hline$D_{4}$ & 21 & 02000040 & $D_{5}+A_{2}$ & 79 & 02002022 \\
\hline $2 A_{2}+2 A_{1}$ & 22 & 10100100 & & 80 & 00400040 \\
\hline$A_{3}+2 A_{1}$ & 23 & 10010011 & & 81 & 20200220 \\
\hline \multirow[t]{2}{*}{$D_{4}\left(a_{1}\right)+A_{1}$} & 24 & 11001010 & $E_{6}$ & 82 & 04020240 \\
\hline & 25 & 00100101 & $D_{6}$ & 83 & 21031031 \\
\hline \multirow[t]{3}{*}{$A_{3}+A_{2}$} & 26 & 20100011 & $D_{7}\left(a_{2}\right)$ & 84 & 31010211 \\
\hline & 27 & 10001002 & & 85 & 11111111 \\
\hline & 28 & 01010100 & $A_{7}$ & 86 & 12111111 \\
\hline \multirow[t]{2}{*}{$A_{4}$} & 29 & 02020000 & $E_{6}\left(a_{1}\right)+A_{1}$ & 87 & 13111101 \\
\hline & 30 & 00020020 & & 88 & 11111121 \\
\hline \multirow[t]{2}{*}{$A_{3}+A_{2}+A_{1}$} & 31 & 00100003 & $E_{7}\left(a_{3}\right)$ & 89 & 11121121 \\
\hline & 32 & 10101001 & & 90 & 30130130 \\
\hline$D_{4}+A_{1}$ & 33 & 11001030 & $E_{8}\left(b_{2}\right)$ & 91 & 20202022 \\
\hline \multirow[t]{3}{*}{$D_{4}\left(a_{1}\right)+A_{2}$} & 34 & 00000004 & & 92 & 04004000 \\
\hline & 35 & 20002000 & $D_{7}\left(a_{1}\right)$ & 93 & 02022022 \\
\hline & 36 & 00200002 & & 94 & 40040040 \\
\hline \multirow[t]{2}{*}{$A_{4}+A_{1}$} & 37 & 11110010 & & 95 & 20220220 \\
\hline & 38 & 01010110 & $E_{6}+A_{1}$ & 96 & 13111141 \\
\hline $2 A_{3}$ & 39 & 10110100 & $E_{7}\left(a_{2}\right)$ & 97 & 13103041 \\
\hline \multirow[t]{2}{*}{$D_{5}\left(a_{1}\right)$} & 40 & 20100031 & $E_{8}\left(a_{6}\right)$ & 98 & 00400400 \\
\hline & 41 & 01010120 & & 99 & 22202022 \\
\hline \multirow[t]{3}{*}{$A_{4}+2 A_{1}$} & 42 & 21010100 & $D_{7}$ & 100 & 31131211 \\
\hline & 43 & 01200100 & $E_{8}\left(b_{5}\right)$ & 101 & 22202042 \\
\hline & 44 & 10101011 & & 102 & 04004040 \\
\hline
\end{tabular}


TABLE 19. (continued)

\begin{tabular}{|lrl|lll|}
\hline B-C symbol & No. & Labels & B-C symbol & No. & Labels \\
\hline$A_{4}+A_{2}$ & 45 & 00400000 & $E_{7}\left(a_{1}\right)$ & 103 & 13131043 \\
& 46 & 02000200 & $E_{8}\left(a_{5}\right)$ & 104 & 22222022 \\
$A_{5}$ & 47 & 01020110 & & 105 & 40040400 \\
$D_{5}\left(a_{1}\right)+A_{1}$ & 48 & 30001030 & $E_{8}\left(b_{4}\right)$ & 106 & 22222042 \\
& 49 & 10101021 & & 107 & 04040044 \\
$A_{4}+A_{2}+A_{1}$ & 50 & 11010101 & $E_{7}$ & 108 & 34131341 \\
$D_{4}+A_{2}$ & 51 & 40000040 & $E_{8}\left(a_{4}\right)$ & 109 & 22222222 \\
& 52 & 00200022 & & 110 & 44040400 \\
& 53 & 20002020 & $E_{8}\left(a_{3}\right)$ & 111 & 24222242 \\
$E_{6}\left(a_{3}\right)$ & 54 & 02020020 & & 112 & 44040440 \\
& 55 & 00020200 & $E_{8}\left(a_{2}\right)$ & 113 & 44040440 \\
$D_{5}$ & 56 & 02020040 & $E_{8}\left(a_{1}\right)$ & 114 & 44440444 \\
$A_{4}+A_{3}$ & 57 & 11101011 & $E_{8}$ & 115 & 84444444 \\
$A_{5}+A_{1}$ & 58 & 10111011 & & & \\
\hline
\end{tabular}

TABLE 20. Nonzero nilpotent $K^{c}$-orbits in $\mathfrak{p}^{c}$ for $\mathfrak{g}$ of type EIX

\begin{tabular}{|lrl|lrl|}
\hline B-C symbol & No. & Labels & B-C symbol & No. & Labels \\
\hline$A_{1}$ & 1 & 00000010 & $D_{4}\left(a_{1}\right)$ & 19 & 00000040 \\
$2 A_{1}$ & 2 & 10000002 & & 20 & 20000022 \\
& 3 & 00000100 & $D_{4}$ & 21 & 00000048 \\
$3 A_{1}$ & 4 & 00000013 & & 22 & 20000044 \\
& 5 & 10000011 & $A_{3}+A_{2}$ & 23 & 01100012 \\
$A_{2}$ & 6 & 00000004 & $A_{4}$ & 24 & 40000004 \\
& 7 & 00000022 & & 25 & 20000200 \\
& 8 & 20000002 & $A_{4}+A_{1}$ & 26 & 10100111 \\
$A_{2}+A_{1}$ & 9 & 11000001 & $D_{5}\left(a_{1}\right)$ & 27 & 01100034 \\
$A_{2}+2 A_{1}$ & 10 & 10000102 & $A_{4}+A_{2}$ & 28 & 00020000 \\
& 11 & 00010000 & $A_{5}$ & 29 & 10000313 \\
$A_{3}$ & 12 & 10000024 & $E_{6}\left(a_{3}\right)$ & 30 & 20000222 \\
& 13 & 00000122 & & 31 & 00000404 \\
$2 A_{2}$ & 14 & 00000200 & $D_{5}$ & 32 & 20000244 \\
$2 A_{2}+A_{1}$ & 15 & 10000111 & & 33 & 40000048 \\
$A_{3}+A_{1}$ & 16 & 10000113 & $A_{6}$ & 34 & 00020200 \\
& 17 & 10000031 & $E_{6}\left(a_{1}\right)$ & 35 & 40000404 \\
$D_{4}\left(a_{1}\right)$ & 18 & 00000204 & $E_{6}$ & 36 & 40000448 \\
\hline
\end{tabular}

\section{REFERENCES}

[1] D. Barbasch and M.R. Sepanski, Closure ordering and the Kostant-Sekiguchi correspondence, Proc. Amer. Math. Soc. 126 (1998), pp. 311-317.

[2] M. Berger, Les espaces symétriques non compacts, Ann. Sci. École Norm. Sup., 74 (1957), pp. 85-177.

[3] N. Bourbaki, Groupes et algèbres de Lie, Chapitres 7 et 8, Hermann, Paris, 1975.

[4] D.H. Collingwood And W.M. McGovern, Nilpotent Orbits in Semisimple Lie Algebras, Van Nostrand Reinhold, New York, 1993.

[5] D.Ž. Đoković, Classification of nilpotent elements in simple exceptional real Lie algebras of inner type and description of their centralizers, J. Algebra, 112 (1988), pp. 503-524. 
[6] ___ Classification of nilpotent elements in simple real Lie algebras $E_{6(6)}$ and $E_{6(-26)}$ and description of their centralizers, J. Algebra, 116 (1988), pp. 196-207.

[7] _ _ The closure diagrams for nilpotent orbits of the real forms $E$ VI and E VII of $E_{7}$, Represent. Theory, 5 (2001), pp. 17-42. Correction: ibid, p. 503.

[8] _. The closure diagram for nilpotent orbits of the real form EIX of $E_{8}$, Asian J. Math., 5 (2001), no. 3, pp. 561-584.

[9] D.Ž. ĐoKović, N. LEMIRE, AND J. SEKIGUCHI, The closure ordering of adjoint nilpotent orbits in $\mathfrak{5 0}(p, q)$, Tohoku Math. J. (2), 53 (2001), no. 3, pp. 395-442.

[10] J. SEKIGUCHI, Remarks on nilpotent orbits of a symmetric pair, J. Math. Soc. Japan, 39 (1987), pp. $127-138$. 
